\title{
Vacuum metastability with black holes
}

\author{
Philipp Burda, ${ }^{a, 1}$ Ruth Gregory ${ }^{a, b}$ annd Ian G. Moss ${ }^{c}$ \\ ${ }^{a}$ Centre for Particle Theory, Durham University, \\ South Road, Durham, DH1 3LE, U.K. \\ ${ }^{b}$ Perimeter Institute, 31 Caroline Street North, \\ Waterloo, ON, N2L 2Y5, Canada \\ ${ }^{c}$ School of Mathematics and Statistics, Newcastle University, \\ Newcastle Upon Tyne, NE1 7RU, U.K. \\ E-mail: philipp.burda@durham.ac.uk, r.a.w.gregory@durham.ac.uk, \\ ian.moss@newcastle.ac.uk
}

ABSTRACT: We consider the possibility that small black holes can act as nucleation seeds for the decay of a metastable vacuum, focussing particularly on the Higgs potential. Using a thin-wall bubble approximation for the nucleation process, which is possible when generic quantum gravity corrections are added to the Higgs potential, we show that primordial black holes can stimulate vacuum decay. We demonstrate that for suitable parameter ranges, the vacuum decay process dominates over the Hawking evaporation process. Finally, we comment on the application of these results to vacuum decay seeded by black holes produced in particle collisions.

KEYWords: Solitons Monopoles and Instantons, Black Holes

ARXiv EPrint: 1503.07331

\footnotetext{
${ }^{1}$ On leave of absence from ITEP, Moscow.
} 


\section{Contents}

1 Introduction 1

2 Thin-wall bubbles $\quad 4$

2.1 Constructing the instanton 4

2.2 Computing the action 6

3 Instanton solutions $\quad 8$

3.1 Coleman de Luccia 9

3.2 The general instanton 9

$\begin{array}{ll}3.3 \text { Charged black hole instantons } & 12\end{array}$

4 Application to the Higgs vacuum. 14

$\begin{array}{lll}5 & \text { Conclusions } & 19\end{array}$

A The limits on $k_{1}, k_{2} \quad 21$

B Alternative bounce action calculation $\quad 23$

$\begin{array}{ll}\text { C Higher dimensional instantons } & 25\end{array}$

\section{Introduction}

We live in a world in which the fundamental properties of matter are manifestly unchanging on the timescale of our everyday lives. Nevertheless, the recent discovery of the Higgs boson $[1,2]$ raises the possibility that, even within the standard model of particle physics, the present vacuum state of the universe may not be stable, but only metastable, with another lower energy state at high expectation values of the Higgs field [3-7]. In general, this would not conflict with observation because the lifetime of the present vacuum would be far longer than the age of the universe. Indeed, the possibility that we live in a metastable state was mooted long before the discovery of the Higgs [8-16].

Investigations of vacuum decay in the context of quantum field theory are usually based on the bubble nucleation arguments of Coleman et al. [17-19], (see also [20]) which relate vacuum decay to the random nucleation of critical bubbles of a new vacuum or phase. However, in many familiar examples of phase transitions beyond the realm of particle physics, the transition is dominated by bubbles which nucleate around fixed sites, usually impurities in the medium or imperfections in a containment vessel. It is therefore important to investigate whether the metastable Higgs vacuum might be ruled out if the seeded nucleation rates for vacuum decay are comparatively large. 
In recent work [21], following earlier work by Hiscock and Berezin [22, 23], we looked at the effect of gravitational inhomogeneities acting as seeds of cosmological phase transitions in de Sitter space. We found that the decay rates were considerably enhanced by the presence of black holes. Following our work, Sasaki and Yeom [24] have investigated the unitarity implications of bubble nucleation in Schwarzschild-Anti de Sitter spacetimes (see also [25] for a discussion of vacuum stability in the early universe). In this paper we extend our previous results [21], to cover all possible gravitational nucleation processes, focussing in particular on the nucleation of bubbles of Anti de Sitter (AdS) spacetime within a vacuum first reported in [26].

We follow the approach of Coleman and de Luccia [19], and assume that the nucleation probability for a bubble of the new phase is given schematically by

$$
\Gamma=A e^{-B},
$$

where $B$ is the action of an imaginary-time solution to the Einstein-Higgs field equations, or instanton, which approaches the false vacuum at large distances. However, unlike Coleman and de Luccia, we consider a spherically symmetric bubble on a black hole background. The nucleation process typically requires an instanton that has a conical singularity at the black hole horizon. Analogous instantons were considered before in [27, 28] and fall within the generalised type introduced by Hawking and Turok [29, 30]. As in our previous paper, we show that the nucleation probability is well-defined. An alternative interpretation of (1.1) and the instanton has been given in [31].

The vacuum decay process is based on a static black hole, in which a bubble nucleates outside the black hole and either completely replaces the black hole with a bubble of true vacuum expanding outwards, or nucleates a static bubble leaving a remnant black hole surrounded by true vacuum. This latter solution is not stable, and small fluctuations will lead it to either expand as with the first situation completing the phase transition, or to collapse back inwards leaving the initial state unchanged. Of course, this description does not explicitly account for any time dependence of the black hole due to Hawking evaporation, however, we can apply the same argument as that employed for black hole particle production, namely, we consider only vacuum decay precesses which have timescales short compared to the evaporation rate. In other words, we have some confidence in our results when the vacuum decay rate exceeds the mass decay rate of the black hole. (The effects of Hawking radiation on tunnelling rates have been investigated in [32, 33]).

We will show that the vacuum decay seeded by black holes greatly exceeds the Hawking evaporation rate for particle physics scale bubbles. This clearly has relevance for the Higgs potential, which we consider explicitly in $\S 4$. A primordial black hole losing mass by the Hawking process would decay down to a mass around 10-100 times the Planck mass and then seed a vacuum transition. The fact that this has not happened therefore means that either the Higgs parameters are not the the relevant range (a small region of parameter space for this purely gravitational argument) or there are no primordial black holes in the observable universe.

Since our main application is to the Higgs vacuum, we will first summarize some of the features of the Higgs potential relevant to the calculation. As with the phenomenological 


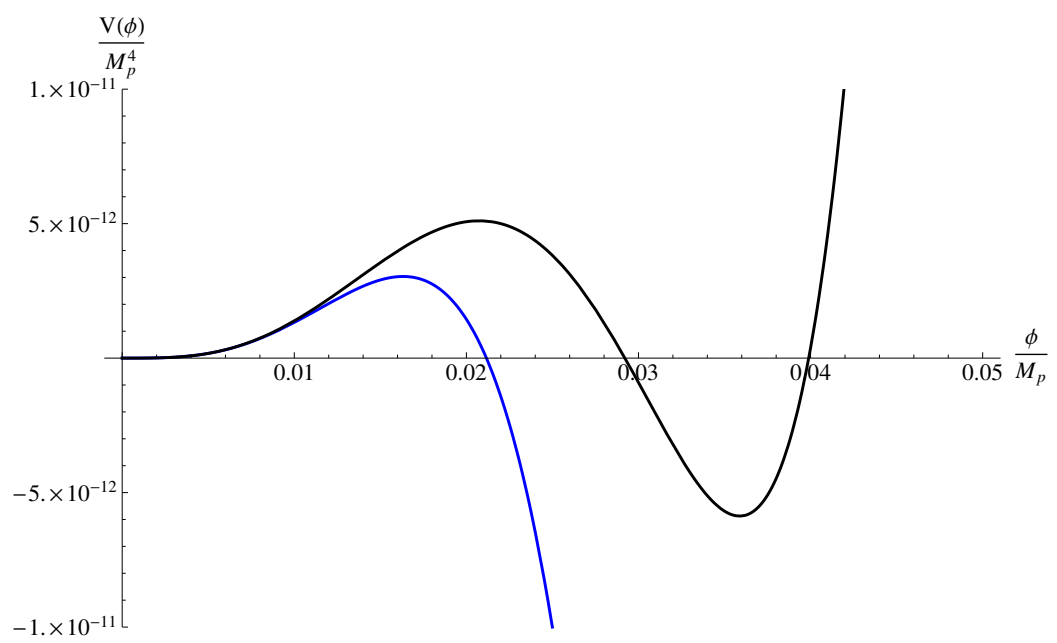

Figure 1. The Higgs potential at large values of one of the Higgs field components $\phi$. The parameter values for the blue line are $\lambda_{*}=-0.001, \phi_{*}=0.5 M_{p}$. The black line shows the effect of adding a $\phi^{6}$ term with coefficient $\lambda_{6}=0.34$.

explorations of the Higgs potential, we write the potential in terms of an overall magnitude of the Higgs, $\phi$, and approximate the potential with an effective coupling $\lambda_{\text {eff }}$,

$$
V(\phi)=\frac{1}{4} \lambda_{\text {eff }}(\phi) \phi^{4}
$$

The exact form of $\lambda_{\text {eff }}$ is determined by a renormalisation group computation with the parameters and masses measured at low-energy. Two-loop calculations of the running coupling [3, 34-36], can be approximated by an expression of the form

$$
\lambda_{\mathrm{eff}} \approx \lambda_{*}+b\left(\ln \frac{\phi}{\phi_{*}}\right)^{2}
$$

where $-0.01 \lesssim \lambda_{*} \lesssim 0,0.1 M_{p} \lesssim \phi_{*} \lesssim M_{p}$ and $b \sim 10^{-4}$. The uncertainty on these parameter ranges is due mostly to experimental uncertainties in the Higgs mass and the top quark mass, however the possibility of negative $\lambda_{\text {eff }}$ approaching the Planck scale is quite real. The present-day broken symmetry vacuum may therefore be a metastable state, but quantum tunnelling in the Higgs potential determined by the usual Coleman de Luccia expressions is very slow, and the lifetime of the false vacuum far exceeds the lifetime of the universe.

The observation of negative $\lambda_{\text {eff }}$ of course assumes no corrections from new physics between the $\mathrm{TeV}$ scale and the Planck scale. We might expect quantum gravity, or other effects will have to be taken into account. On dimensional grounds, we can write modifications to the potential of the following form [37-42],

$$
V(\phi)=\frac{1}{4} \lambda_{\mathrm{eff}}(\phi) \phi^{4}+\frac{1}{4}(\delta \lambda)_{\mathrm{bsm}} \phi^{4}+\frac{1}{6} \lambda_{6} \frac{\phi^{6}}{M_{p}^{2}}+\frac{1}{8} \lambda_{8} \frac{\phi^{8}}{M_{p}^{4}}+\ldots
$$

where $(\delta \lambda)_{\text {bsm }}$ includes corrections from BSM physics, and the polynomial terms represent unknown physics from the Planck scale. If these coefficients are similar in magnitude, then 
the small size of $\lambda_{\text {eff }}$ at the Planck scale has the consequence that there is an intermediate range of $\phi$ where the potential is determined predominantly by $\lambda_{\text {eff }}$ and $\lambda_{6}$.

Quantum tunnelling in a corrected potential has been explored by Branchina et al. [39, 40] (see also [43]). They considered potentials with $\lambda_{*} \sim-0.1$, where the potential barrier occurs at $\phi \ll M_{p}$, and they further enhanced the tunnelling rate by taking $\lambda_{6}=-2$. They claimed a greatly enhanced tunnelling rate, with a lifetime much shorter than the age of the universe, however, their discussion did not include gravitational interactions.

The aim of the present paper is to analyse models which appear stable on cosmological timescales when using the CDL results, but may become unstable due to enhancement of the tunnelling rate by a nucleation seed, which we will take to be a microscopic black hole. For this semi-analytic investigation, we consider the nucleation with thin-wall bubbles of the true vacuum in an analogous way to Coleman and de Luccia. In terms of the Higgs, this thin-wall bubble nucleation requires the potential to be relatively shallow at the true vacuum, and this requires a large positive $\phi^{6}$ term. To go beyond this approximation, which allows us to use pure gravitational arguments, will require a detailed numerical study that we will present in future work.

The outline of the paper is as follows. We first review then extend the thin wall instanton method in $\S 2$, directly calculating the instanton action in the thin wall limit as a function of wall trajectory and black holes masses. In $\S 3$ we describe the solutions for the instantons and discuss the preferred decay process for a general seed mass black hole (including charge). In $\S 4$ we apply the results to the case of the Higgs potential, and present a full comparative calculation with the decay of the black hole due to Hawking radiation. Finally, in $\S 5$, we discuss possible extensions to higher dimensions and collider

black holes. Note we use units in which $\hbar=c=1$, and use the reduced Planck mass $M_{p}^{2}=1 /(8 \pi G)$.

\section{Thin-wall bubbles}

In this section we describe how to construct a thin wall instanton, along the lines of Coleman et al. [17-19], but with the difference that we suppose that an inhomogeneity is present. Complementary to our earlier work [21], we apply Israel's thin wall techniques [44] to the bubble wall, and describe the inhomogeneity by a black hole. (In appendix B we calculate the instanton action for more general inhomogeneous configurations, with the proviso that they be static.)

\subsection{Constructing the instanton}

The physical process of vacuum decay with an inhomogeneity can be represented gravitationally by a Euclidean solution with two 'Schwarzschild' bulks which have different cosmological constants separated by a thin wall with constant tension (for a general proof of this result in the context of braneworlds, see [45, 46]). On each side of the wall the geometry has the form

$$
d s^{2}=f(r) d \tau_{ \pm}^{2}+\frac{d r^{2}}{f(r)}+r^{2} d \Omega_{I I}^{2}, \quad f(r) \equiv 1-\frac{2 G M_{ \pm}}{r}-\frac{\Lambda_{ \pm} r^{2}}{3}
$$


where $\tau_{ \pm}$are the different time coordinates on each side of the wall, and the wall, or boundary of each bulk, is parametrised by some trajectory $r=R(\lambda)$ (the angular $\theta$ and $\phi$ coordinates are the same on each side). The Israel junction conditions [44] relate the solution inside the bubble with mass $M_{-}$and cosmological constant $\Lambda_{-}$, to the solution outside the bubble with mass $M_{+}$and cosmological constant $\Lambda_{+}$. Since the bubble exterior is in the false vacuum, we have $\Lambda_{+}>\Lambda_{-} . \quad\left(\Lambda_{+}<\Lambda_{-}\right.$was discussed by Aguirre and Johnson [47, 48], and the case $M_{-}=0$ has been discussed by Sasaki and Yeom [24]). In general, the bubble will follow a time-dependent trajectory representing a reflection, or bounce.

Following the Israel approach [44], we choose to parametrize the wall trajectory by the proper time of a comoving observer, i.e. $\lambda$ is chosen so that

$$
f \dot{\tau}_{ \pm}^{2}+\frac{\dot{R}^{2}}{f}=1
$$

and take normal forms that point towards increasing $r$ :

$$
n_{ \pm}=\dot{\tau}_{ \pm} d r_{ \pm}-\dot{r} d \tau_{ \pm}
$$

where dots denote derivatives with respect to $\lambda$. We also take $\dot{\tau}_{ \pm} \geq 0$ for orientability (see also [24]). In these conventions, the Israel junction conditions are

$$
f_{+} \dot{t}_{+}-f_{-} \dot{t}_{-}=-4 \pi G \sigma R
$$

The combination of surface tension and Newton's constant recurs so frequently that for clarity we define

$$
\bar{\sigma}=2 \pi G \sigma .
$$

To find solutions to the equations of motion, first note that the junction condition (2.4) implies

$$
f_{ \pm} \dot{\tau}_{ \pm}=\left(f_{ \pm}-\dot{R}^{2}\right)^{1 / 2}=\frac{f_{-}-f_{+}}{4 \bar{\sigma} R} \mp \bar{\sigma} R
$$

It is convenient to rewrite this as an equation for $\dot{R}$ using the explicit forms for $f_{ \pm}$

$$
\dot{R}^{2}=1-\left(\bar{\sigma}^{2}+\frac{\bar{\Lambda}}{3}+\frac{(\Delta \Lambda)^{2}}{144 \bar{\sigma}^{2}}\right) R^{2}-\frac{2 G}{R}\left(\bar{M}+\frac{\Delta M \Delta \Lambda}{24 \bar{\sigma}^{2}}\right)-\frac{(G \Delta M)^{2}}{4 R^{4} \bar{\sigma}^{2}},
$$

where $\Delta M=M_{+}-M_{-}$and $\bar{M}=\left(M_{+}+M_{-}\right) / 2$ with similar expressions for $\Lambda$.

Although this seems to be a more complex system than that considered in [21], in fact it is possible to rescale the variables so that the analysis is very nearly identical to that in [21]. To begin with, define

$$
\ell^{2}=\frac{3}{\Delta \Lambda}, \quad \gamma=\frac{4 \bar{\sigma} \ell^{2}}{1+4 \bar{\sigma}^{2} \ell^{2}}, \quad \alpha^{2}=1+\frac{\Lambda_{-} \gamma^{2}}{3},
$$

and rescale the coordinates to $\tilde{R}=\alpha R / \gamma, \tilde{\tau}=\alpha \tau / \gamma, \tilde{\lambda}=\alpha \lambda / \gamma$. Then writing

$$
k_{1}=\frac{2 \alpha G M_{-}}{\gamma}+\frac{(1-\alpha) \alpha G \Delta M}{\bar{\sigma} \gamma^{2}}, \quad k_{2}=\frac{\alpha^{2} G \Delta M}{2 \bar{\sigma} \gamma^{2}} .
$$


gives a Friedman-like equation for $\tilde{R}(\tilde{\lambda})$ :

$$
\left(\frac{d \tilde{R}}{d \tilde{\lambda}}\right)^{2}=1-\left(\tilde{R}+\frac{k_{2}}{\tilde{R}^{2}}\right)^{2}-\frac{k_{1}}{\tilde{R}}=-U(\tilde{R})
$$

together with equations for $\tilde{\tau}_{ \pm}$(given in appendix A). These equations with $\alpha=1$ are precisely the system explored in [21]. The allowed parameter ranges for $k_{1}$ and $k_{2}$ are obtained similarly, and discussed in appendix A.

\subsection{Computing the action}

To compute the action of the bounce, we need to compute the Euclidean action of the thin wall instanton:

$$
\begin{aligned}
I_{E}= & -\frac{1}{16 \pi G} \int_{\mathcal{M}_{+}} \sqrt{g}\left(\mathcal{R}_{+}-2 \Lambda_{+}\right)-\frac{1}{16 \pi G} \int_{\mathcal{M}_{-}} \sqrt{g}\left(\mathcal{R}_{-}-2 \Lambda_{-}\right) \\
& +\frac{1}{8 \pi G} \int_{\partial \mathcal{M}_{+}} \sqrt{h} K_{+}-\frac{1}{8 \pi G} \int_{\partial \mathcal{M}_{-}} \sqrt{h} K_{-}+\int_{\mathcal{W}} \sigma \sqrt{h}
\end{aligned}
$$

and subtract the action of the background. In this expression, $\partial \mathcal{M}_{ \pm}$refers to the boundary induced by the wall - there may also be additional boundary or bulk terms required for renormalisation of the action (see below). Note that we have reversed the sign of the $\partial \mathcal{M}_{-}$ normal in the Gibbons-Hawking boundary term so that it agrees with the outward pointing normal of the Israel prescription. On each side of the wall in the bulk we have $\mathcal{R}_{ \pm}=4 \Lambda_{ \pm}$, and the Israel equations give $K_{+}-K_{-}=-12 \pi G \sigma$.

There are three parts to the computation of the action, $\mathcal{M}_{-}, \mathcal{M}_{+}$, and $\mathcal{W}$.

- $\mathcal{M}_{-}$: integrating the bulk term for the "-" side of the wall has two contributions, one from the cosmological constant in the bulk volume, and a contribution from any conical deficit at the black hole event horizon, should one exist. A description of how to deal with conical deficits was given in an appendix of [21], essentially the deficit gives a contribution proportional to the horizon area times the deficit angle. Supposing that the periodicity of the Euclidean time coordinate, $\beta$, set by the wall solution, may not be the same as the natural horizon periodicity, $\beta_{-}=$ $4 \pi r_{h} /\left(1-\Lambda_{-} r_{h}^{2}\right)$, this gives a contribution to the action from $\mathcal{M}_{-}$of:

$$
\begin{aligned}
I_{\mathcal{M}_{-}} & =-\frac{\beta_{-}-\beta}{\beta_{-}} \frac{\mathcal{A}_{-}}{4 G}-\frac{1}{4 G} \int \frac{2 \Lambda_{-}}{3}\left(R^{3}-r_{h}^{3}\right) d \tau_{-} \\
& =-\frac{\mathcal{A}_{-}}{4 G}+\frac{\beta}{4 G}\left[\frac{\mathcal{A}_{-}}{\beta_{-}}+\frac{2 \Lambda_{-} r_{h}^{3}}{3}-2 G M_{-}\right]+\frac{1}{4 G} \int d \lambda R^{2} f_{-}^{\prime} \dot{\tau}_{-}
\end{aligned}
$$

where $\mathcal{A}_{-}$is the area of the black hole horizon in $\mathcal{M}_{-}$. Inserting the value of $\beta_{-}$, and taking into account the value of $r_{h}$, the term in square brackets is identically zero, and this contribution to the action does not explicitly depend on the periodicity or indeed any conical deficit angle. 
- $\mathcal{M}_{+}$: the computation of the action of $\mathcal{M}_{+}$is a little more involved, as different regularisation prescriptions are needed for the different asymptotics of (A)dS or flat spacetime.

For Schwarzschild de Sitter, the radial coordinate in the static patch has a finite range, and terminates at the cosmological horizon $r_{c}$, which has a natural periodicity $\beta_{c}=-4 \pi r_{c}^{2} /\left(2 G M_{+}-2 \Lambda_{+} r_{c}^{3} / 3\right)$.

$$
\begin{aligned}
I_{\mathcal{M}_{+}} & =-\frac{\left(\beta_{c}-\beta\right) \mathcal{A}_{c}}{4 G \beta_{c}}-\frac{1}{4 G} \int \frac{2 \Lambda_{+}}{3}\left(r_{c}^{3}-R^{3}\right) d \tau_{+} \\
& =-\frac{\mathcal{A}_{c}}{4 G}+\frac{\beta}{4 G}\left[\frac{\mathcal{A}_{c}}{\beta_{c}}-\frac{2 \Lambda_{+} r_{c}^{3}}{3}+2 G M_{+}\right]-\frac{1}{4 G} \int d \lambda R^{2} f_{+}^{\prime} \dot{\tau}_{+}
\end{aligned}
$$

where $\mathcal{A}_{c}$ is the area of the cosmological event horizon. Once again, substituting the values of $\beta_{c}$ and $r_{c}$ demonstrates that the bracketed term vanishes. For future reference, we note the value of the background SDS action at arbitrary periodicity derived in [21]:

$$
I_{E S D S}=-\frac{\mathcal{A}_{c}}{4 G}-\frac{\mathcal{A}_{+}}{4 G}
$$

where $\mathcal{A}_{+}$is the horizon area of the black hole of mass $M_{+}$. Note that this expression is $\beta$-independent as discussed in [21].

For Schwarzschild (and Schwarzschild-AdS) the range of the radial coordinate is now infinite, and we must perform a renormalization procedure. For Schwarzschild, there is no contribution from the bulk integral, and instead we consider an artificial boundary at large $r_{0}$, with a subtracted Gibbons-Hawking term so that flat space has zero action [49].

$$
I_{\mathcal{M}_{+}}=\frac{1}{8 \pi G} \int_{r=r_{0}} \sqrt{h}\left(K-K_{0}\right)=\frac{\beta M_{+}}{2}=\beta M_{+}-\frac{1}{4 G} \int d \lambda f_{+}^{\prime} R^{2} \dot{\tau}_{+}
$$

Again for future reference, computing the background Euclidean Schwarzschild action at arbitrary periodicity (with the same background subtraction prescription) yields

$$
I_{E S C H}=-\frac{\mathcal{A}_{+}}{4 G}+\beta M_{+}
$$

inputting the value of $\beta_{S C H}=8 \pi G M_{+}$.

For AdS on the other hand, we must subtract off the divergent volume contribution [50] by again introducing a fiducial boundary at $r_{0}$, and subtracting a pure AdS integral, which must have an adjusted time-periodicity so that the boundary manifolds at $r_{0}$ agree:

$$
\beta_{0}=\beta \frac{f_{+}^{1 / 2}}{\left(1-\Lambda_{+} r_{0}^{2} / 3\right)^{1 / 2}} \simeq\left(1+\frac{3 G M_{+}}{r_{0}^{3} \Lambda}\right) \beta .
$$

Thus

$$
\begin{aligned}
I_{\mathcal{M}_{+}} & =-\frac{1}{4 G} \int d \tau \frac{2 \Lambda_{+}}{3}\left(r_{0}^{3}-R^{3}\right)+\frac{1}{4 G} \int d \tau_{0} \frac{2 \Lambda_{+}}{3} r_{0}^{3} \\
& =\beta M_{+}-\frac{1}{4 G} \int d \lambda f_{+}^{\prime} R^{2} \dot{\tau}_{+}
\end{aligned}
$$


i.e. an identical result to the Schwarzschild case (2.15). Computing the background Euclidean Schwarzschild-AdS action at arbitrary periodicity we get

$$
I_{E S A D S}=-\frac{\mathcal{A}_{+}}{4 G}+\beta M_{+}
$$

again, the same expression as for Schwarzschild, (2.16).

- $\mathcal{W}$ : finally, the contribution to the action from the wall has a particularly simple form as the Gibbons-Hawking boundary terms from the wall come in the combination of the Israel junction conditions. We therefore obtain

$$
I_{\mathcal{W}}= \pm \frac{1}{8 \pi G} \int_{\partial \mathcal{M}_{ \pm}} \sqrt{h} K+\int_{\mathcal{W}} \sigma \sqrt{h}=-\int_{\mathcal{W}} \frac{\sigma}{2} \sqrt{h}=\frac{1}{2 G} \int d \lambda R\left(f_{+} \dot{\tau}_{+}-f_{-} \dot{\tau}_{-}\right)
$$

having used $f_{+} \dot{\tau}_{+}-f_{-} \dot{\tau}_{-}=-2 \bar{\sigma} R$.

Putting all of these results together, we find that the action of the instanton solution is

$$
I_{E}=-\frac{\mathcal{A}_{-}}{4 G}+\frac{1}{2 G} \int d \lambda\left[\left(R-3 G M_{+}\right) \dot{\tau}_{+}-\left(R-3 G M_{-}\right) \dot{\tau}_{-}\right]+\left\{\begin{array}{cc}
\beta M_{+} & \Lambda_{+} \leq 0 \\
-\frac{\mathcal{A}_{c}}{4 G} & \Lambda_{+}>0
\end{array}\right.
$$

Thus the bounce action, given by subtracting the background Schwarzschild/S(A)dS action is:

$$
B=\frac{\mathcal{A}_{+}}{4 G}-\frac{\mathcal{A}_{-}}{4 G}+\frac{1}{4 G} \oint d \lambda\left\{\left(2 R f_{+}-R^{2} f_{+}^{\prime}\right) \dot{\tau}_{+}-\left(2 R f_{-}-R^{2} f_{-}^{\prime}\right) \dot{\tau}_{-}\right\}
$$

This expression is the central result of this section, and is independent of any choice of periodicity of Euclidean time, and independent of the choices of cosmological constant on each side of the wall. It is in fact even valid when the black hole is charged, as we will consider in the next section.

\section{Instanton solutions}

In the previous section we derived the equations of motion for a bubble wall separating a region of true vacuum from the false vacuum, and derived the "master expression" (2.22) for the instanton action. In this section we discuss general properties of these solutions, and demonstrate how the action varies as we change the seed black hole mass and the wall tension. Rather than presenting absolute values of the bounce action, it proves useful instead to present a comparator to the 'Coleman de Luccia' action, by which we mean the bounce solution in the absence of any black holes (but with, for now, arbitrary cosmological constants). 


\subsection{Coleman de Luccia}

The 'CDL' bubble wall satisfies (2.10), (A.1), and (A.2), which are solved by

$$
\begin{aligned}
\tilde{R} & =\cos \tilde{\lambda} \\
\tilde{t}_{-} & =\frac{\alpha}{\sqrt{\alpha^{2}-1}} \arctan \sqrt{\alpha^{2}-1} \sin \tilde{\lambda} ; \\
\tilde{t}_{+} & =\frac{\alpha}{\sqrt{\alpha^{2}-(1-2 \bar{\sigma} \gamma)^{2}}} \arctan \frac{\sqrt{\alpha^{2}-(1-2 \bar{\sigma} \gamma)^{2}}}{(1-2 \bar{\sigma} \gamma)} \sin \tilde{\lambda}
\end{aligned}
$$

(where $\tilde{\lambda} \in\left[-\frac{\pi}{2}, \frac{\pi}{2}\right]$ for the full bounce) and the action can be computed analytically as

$$
B_{C D L}=-\frac{1}{2 G} \int R\left(\dot{\tau}_{+}-\dot{\tau}_{-}\right)=\frac{\pi}{G} \frac{\bar{\sigma} \gamma^{3}}{\alpha(\alpha+1)(\alpha+1-2 \bar{\sigma} \gamma)} \stackrel{\Lambda_{+}=0}{\longrightarrow} \frac{\pi \ell^{2}}{G} \frac{16(\bar{\sigma} \ell)^{4}}{\left(1-4 \bar{\sigma}^{2} \ell^{2}\right)^{2}}
$$

Note that by analytic continuation, these expressions include arbitrary $\Lambda_{ \pm}$, for which $\alpha<1$ or $1-2 \bar{\sigma} \gamma$ are possible. In this special case the symmetry of the bubble solution has been raised from $O(3)$ to $O(4)$, and the result for the tunnelling rate agrees with explicitly $O(4)$ symmetric methods.

\subsection{The general instanton}

The general bubble wall will have a black hole mass term on each side, and a general instanton will consist of a bubble trajectory between a minimum and maximum value of $\tilde{R}$. For fixed seed mass, $M_{+}$, there will be a range of allowed $k_{1}$ and $k_{2}$ (see (A.5)), and a corresponding range of values for the bounce action. By exploring the $\left\{k_{1}, k_{2}\right\}$ parameter space numerically and plotting the ratio of the bounce action to the CDL action, we can build up a qualitative understanding of the preferred instanton for vacuum decay.

For example, if $\Lambda_{+}=0, G M_{+}=\gamma k_{1} / 2 \alpha$, and $G M_{-}=G M_{+}-\gamma k_{2}(1-\alpha) / \alpha^{2}$. Referring to figure 9 , we see there are two possibilities for the range of $k_{2}$, which is now a horizontal line in the $k_{1}$ plot: either the maximal value of $k_{2}$ lies on the $k_{1}^{m}$ branch with $G M_{-}=0$, or on the static branch $k_{1}^{*}\left(k_{2}\right)$. The picture is similar for general $\Lambda_{+}$, but the constant $G M_{+}$ lines are now at an angle, and interpolate between the $k_{1}^{m}$ curve at negative $k_{2}$ and either the $k_{1}^{m}$ line at positive $k_{2}$ or the $k_{1}^{*}\left(k_{2}\right)$ curve. The crossover between the two possibilities occurs at $M_{+}=M_{C}$, given by the algebraic solution to

$$
k_{1}^{*}\left(k_{2}\right)=\frac{2 k_{2}}{\alpha}(1-\alpha)
$$

when we have a static bubble with $G M_{-}=0$. In either case, as $k_{2}$ drops, $G M_{-}$increases until the lower limit of $k_{2}$ is reached at negative $k_{2}$ on the $k_{1}^{m}\left(k_{2}\right)$ curve. By solving numerically for the wall trajectories we find that the action increases as $k_{2}$ drops. The preferred instanton therefore is the one with the maximally allowed value of $k_{2}$ consistent with the value of $G M_{+}$.

This qualitative picture remains true irrespective of the values of $\Lambda_{ \pm}$: for seed mass $M_{+}<M_{C}$, the dominant tunnelling process leaves behind a true vacuum region and removes the black hole. The tunnelling rate is always faster than the vacuum tunnelling rate 


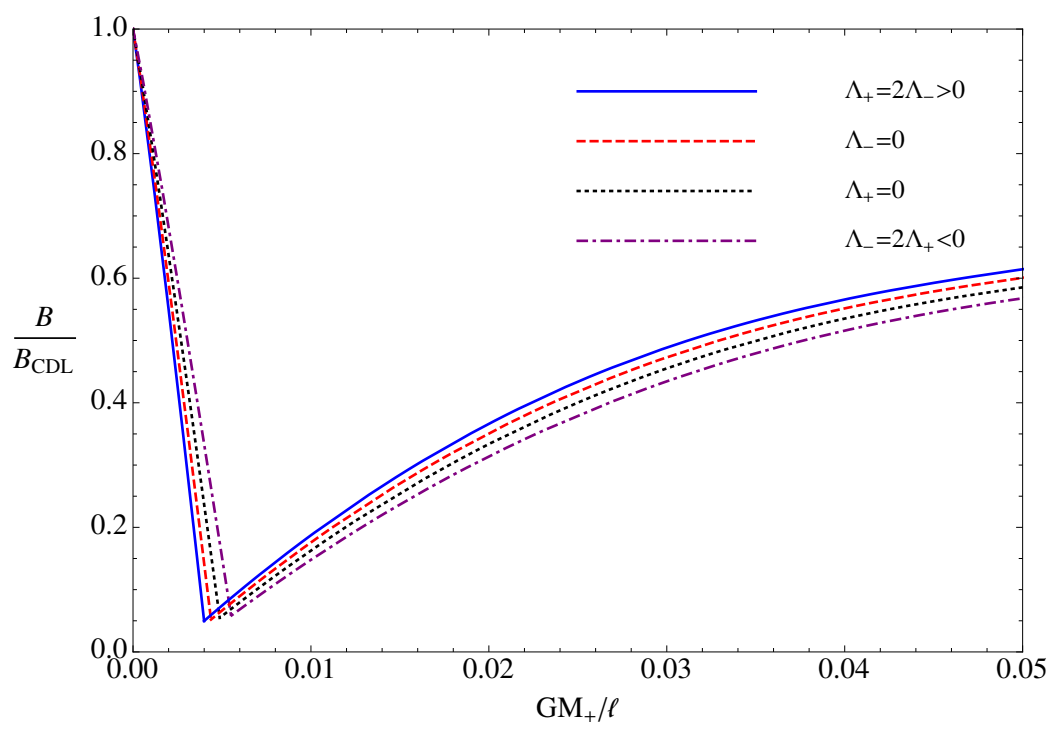

Figure 2. A plot of the minimum bounce action as $M_{+}$is varied for $\bar{\sigma} \ell=0.1$, and varying values of $\Lambda_{+}=6 / \ell^{2}, 3 / \ell^{2}, 0,-3 / \ell^{2}, \Lambda_{-}=3 / \ell^{2}, 0,-3 / \ell^{2},-6 / \ell^{2}$ as indicated. The ratio of the bounce action to the CDL value is plotted, but as $\Lambda_{ \pm}$vary, this value itself changes. For $\bar{\sigma} \ell=0.1, B_{C D L}=$ $0.101,0.117,0.137,0.165 \ell^{2} / L_{p}^{2}$ as $\Lambda_{+}$drops from its maximal to minimal value considered here.

for these instantons. The bounce action reaches a minimum at $M=M_{C}$, where the bubble is static. For $M>M_{C}$ the dominant tunnelling process is a static bubble with a remnant black hole being left behind. As the seed mass increases further, eventually the tunnelling rate becomes lower than the vacuum tunnelling rate. Exploring the instantons for general $\Lambda$ 's, we find that the ratio of $B / B_{C D L}$ changes very little as the $\Lambda$ 's vary. In figure 2 , we show how this dominant tunneling action varies as the values for the cosmological constants are changed. Since the change in $B / B_{C D L}$ is minimal (and $B_{C D L}$ itself is not varying much), we now restrict our discussion to the $\Lambda_{+}=0$ set-up where $\alpha=1-2 \bar{\sigma} \gamma$, and many of the formulae simplify.

Before discussing the general dominant tunneling process, we begin by considering the critical instanton where the static bubble tunnels and removes the seed black hole altogether. Although (3.3) in general is a complicated algebraic equation, for small $\bar{\sigma} \ell$ the various parameters can be expanded straightforwardly to give

$$
k_{1 C} \simeq \frac{64}{27}(\bar{\sigma} \ell)^{2}=\frac{4}{9}-3 k_{2 C} \quad \Rightarrow \quad \frac{G M_{C}}{\ell} \simeq \frac{128}{27}(\bar{\sigma} \ell)^{3}
$$

From (2.22), the action of a static bounce in general is

$$
B=\frac{\mathcal{A}_{+}}{4 G}-\frac{\mathcal{A}_{-}}{4 G}=4 \pi G M_{+}^{2}-\pi G\left(\frac{\ell}{G}\right)^{4 / 3}\left(\mu_{+}^{1 / 3}-\mu_{-}^{1 / 3}\right)^{2}
$$

where

$$
G \mu_{ \pm}=\sqrt{G^{2} M_{-}^{2}+\frac{\ell^{2}}{27}} \pm \frac{G M_{-}}{\ell}
$$




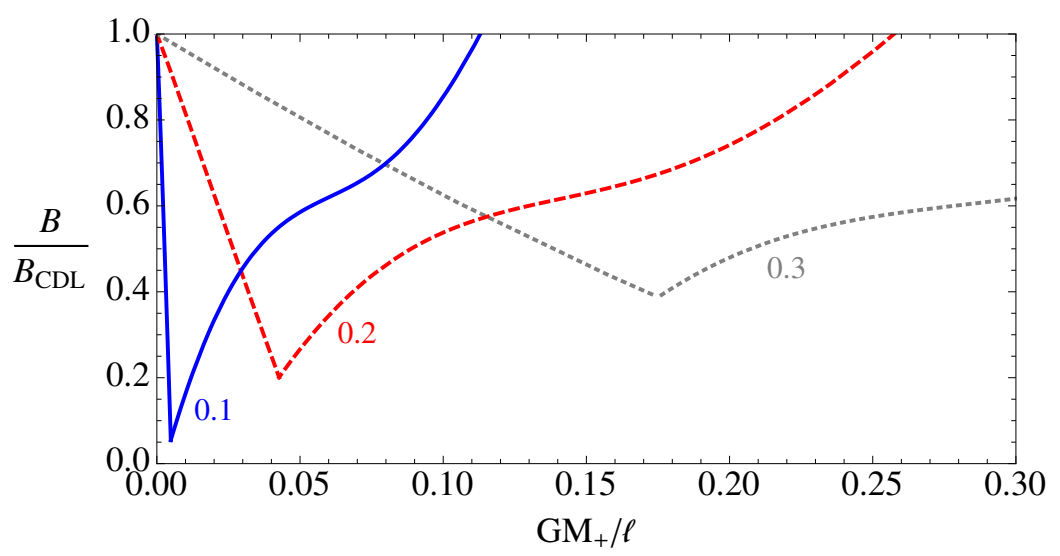

Figure 3. The exponent $B$ for the dominant tunnelling process divided by the appropriate vacuum tunnelling value $B_{C D L}$, for different masses $M_{+}$of the nucleation seed. The surface tension $\sigma$ and AdS radius $\ell$ enter in the combination $\bar{\sigma} \ell$.

although it must be noted that, for the static bubble $M_{-}$is a complicated function of $M_{+}$. For the critical bubble, $G M_{-}=0$, hence the critical bounce action is

$$
B_{C}=4 \pi G M_{C}^{2} \simeq \frac{\pi \ell^{2}}{G}\left(\frac{256}{27}\right)^{2}(\bar{\sigma} \ell)^{6} \simeq\left(\frac{4}{3}\right)^{6}(\bar{\sigma} \ell)^{2} B_{C D L}
$$

Thus as $\bar{\sigma} \ell \rightarrow 0$, the tunnelling action becomes small compared to the CDL action.

One problem with having a small critical mass is of course that the decay rate due to tunnelling may be outstripped by the evaporation rate of the black hole, as we will discuss later, however, what this expansion indicates is that the minimal bounce action for a particular $\bar{\sigma} \ell$ can be extremely small, so that even if we are above the critical black hole mass, the decay rate can still be significant.

Having determined that the dominant tunneling process is either the static bubble or the $G M_{-}=0$ branch, we can now compute the dominant bounce action either by numerically solving the time-dependent bubbles with $G M_{-}=0$, or computing the static bubble actions with $k_{1}=k_{1}^{*}$. We used a simple mathematica program to calculate these exponents, and double checked by a totally numerical computation. The results for some sample values of $\bar{\sigma} \ell$ are presented in figure 3 .

The general bubble solution for $G M_{-}=0$ oscillates between two values $\tilde{R}_{M A X}$ and $\tilde{R}_{M I N}$ where the potential $U(\tilde{R})$ vanishes. This periodic solution in $\tilde{\lambda}$ can only be singlevalued in $\mathcal{M}_{ \pm}$if the manifolds on each side have the same time-periodicity as the bubble wall solution. In general, this will not be the same as the natural periodicity $\Delta \tau_{+}=8 \pi G M_{+}$ of the Euclidean Schwarzschild solution, hence the need to consider general periodicity in the computation of the action in the previous section. For the static solution of course, this is not an issue. The values of $\tilde{R}_{M A X} / \tilde{R}_{M I N}$ are well outside the black hole horizon radius, and move together as $G M_{+}$is increased. Eventually, at $G M_{C}$, the two roots of $U$ meet, and the static branch begins.

The static branch is the preferred instanton with nonzero $G M_{-}$, i.e. with a black hole remnant, although non-static solutions exist with higher action and remnant mass. Initially, 


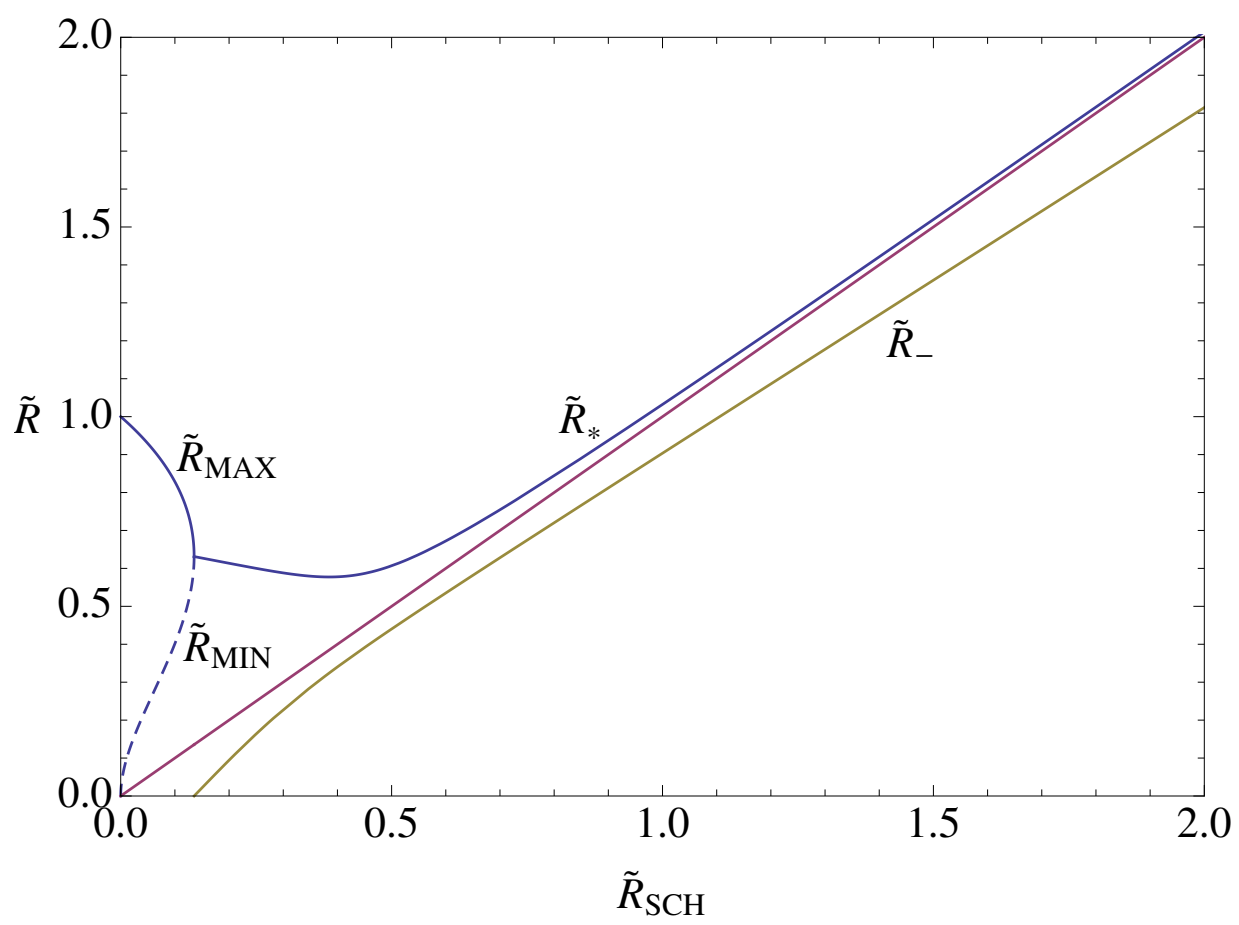

Figure 4. A plot of the variation of the bubble wall radius $\tilde{R}$ as $G M_{+}$is increased for $\bar{\sigma} \ell=0.25$ (chosen to highlight the qualitative features). As $\bar{\sigma} \ell$ drops, the features of the phase diagram remain the same, but 'squash up' towards smaller $\tilde{R}_{S C H}$. The unlabelled red line running from corner to corner represents $\tilde{R}_{S C H}$, the seed black hole horizon radius.

the static bubble shrinks with increasing $G M_{+}$, but remains well outside the Schwarzschild radius, however, as we increase $G M_{+}$further, the bubble becomes constrained by the expanding black hole horizon, and becomes stretched just outside $\tilde{R}_{S C H}$. Meanwhile, the remnant black hole mass $G M_{-}$increases along the static branch and eventually becomes larger than $G M_{+}$, however, because of the negative cosmological constant, the horizon radius, while increasing, does not increase as rapidly as $\tilde{R}_{S C H}$. The static bubble action therefore increases as $G M_{+}$increases, eventually becoming larger than $B_{C D L}$ (see figure 3). Figure 4 illustrates the behaviour of these minimal/maximal and static values of $\tilde{R}$ as $\tilde{R}_{S C H}=2 G M_{+}$varies, the remnant horizon radius is also shown.

\subsection{Charged black hole instantons}

Finally, before considering the case of the Higgs vacuum in detail, we conclude this section by commenting briefly on an obvious generalisation of our instantons to Einstein-YangMills-Higgs theory. The combination of Einstein gravity with Yang-Mills and Higgs fields admits the possibility of charged black hole solutions [51, 52]. Electrically charged black holes can discharge by the emission of charged particles [53], but magnetically charged black holes may be the lightest magnetically charged particles in the theory, in which case a large mass black hole evaporates towards the extremal limit, and the Hawking radiation flux falls to zero. 
Magnetically charged black holes may be produced in the early universe [27, 28], and form the seeds for vacuum decay of an unstable standard model Higgs field. Uncharged black holes can easily evaporate before they seed a phase transition, but the charged black holes hang around for a longer time making them better candidates for vacuum decay nucleation sites.

An $\mathrm{SU}(2) \times \mathrm{U}(1)$ Yang-Mills theory with Higgs field $\mathcal{H}$ in the fundamental $\mathrm{SU}(2)$ representation has no flat-space monopole solutions, but it does have Dirac and Yang-Mills black-hole monoples. The non-abelian monopoles can be constructed from the SU(2) fields $W$ using an ansatz

$$
\begin{aligned}
\mathcal{H} & =\phi(r) \sigma_{r} \mathcal{H}_{0}, \\
W & =G^{1 / 2} \frac{P}{r}\left(\sigma_{\phi} d \theta-\sigma_{\theta} \sin \theta d \phi\right),
\end{aligned}
$$

where $\sigma_{r}, \sigma_{\theta}$ and $\sigma_{\phi}$ are Pauli matrices projected along the spherical polar co-ordinate frame and $\mathcal{H}_{0}$ is constant. (The magnetic charge has been scaled so that an extreme black hole has $P=M$ in the absence of a cosmological constant.)

For a potential which allows decay from flat space to AdS, there are thin-wall bubble solutions with spherical symmetry and constant values of $\phi$ at the appropriate minima of the potential. The metric coefficients are

$$
\begin{aligned}
& f_{-}=1-\frac{2 G M_{-}}{r}+\frac{r^{2}}{\ell^{2}}+\frac{G^{2} P^{2}}{r^{2}}, \\
& f_{+}=1-\frac{2 G M_{+}}{r}+\frac{G^{2} P^{2}}{r^{2}}
\end{aligned}
$$

In this case, the bubble wall carries no magnetic charge. Generalised solutions may also be possible in which the interior and exterior have different magnetic charges.

The action for the bubble solutions is given by the same formula, (2.22), as in the uncharged case, though with the appropriate expressions for $f_{ \pm}$. The plot of the dependence of the action on $G M_{+} / \ell$ is surprisingly similar to the uncharged case at fixed ratio $P / M_{+}$, with one small modification. The time-dependent tunneling solutions prior to the switching on of the static bubbles now do not remove the black hole altogether as this would leave a naked singularity. Instead, the bubbles leave behind an extremal remnant, $M_{-}=M_{\text {ext }}(P)$, where

$$
G M_{\mathrm{ext}}(P)=\frac{\ell}{3 \sqrt{6}}\left(2+\sqrt{1+\frac{12 G^{2} P^{2}}{\ell^{2}}}\right) \sqrt{\sqrt{1+\frac{12 G^{2} P^{2}}{\ell^{2}}}-1} .
$$

The static branch meets this time-dependent branch at a critical mass $M_{C P}$, where the static bubble now has an extremal black hole in its interior. On the static branch, the action is, as before, the difference of the areas of the seed and remnant black holes, but as the extremal limit is approached, the horizon radius of the remnant black hole shrinks only as the root of $M_{+}-M_{C P}$, whereas the radius of the seed black hole (which is not approaching an extremal limit) depends linearly on $M_{+}-M_{C P}$, thus, as we increase $M_{+}$ from $M_{C P}$, the action actually starts to drop briefly, before the effect of the increasing 


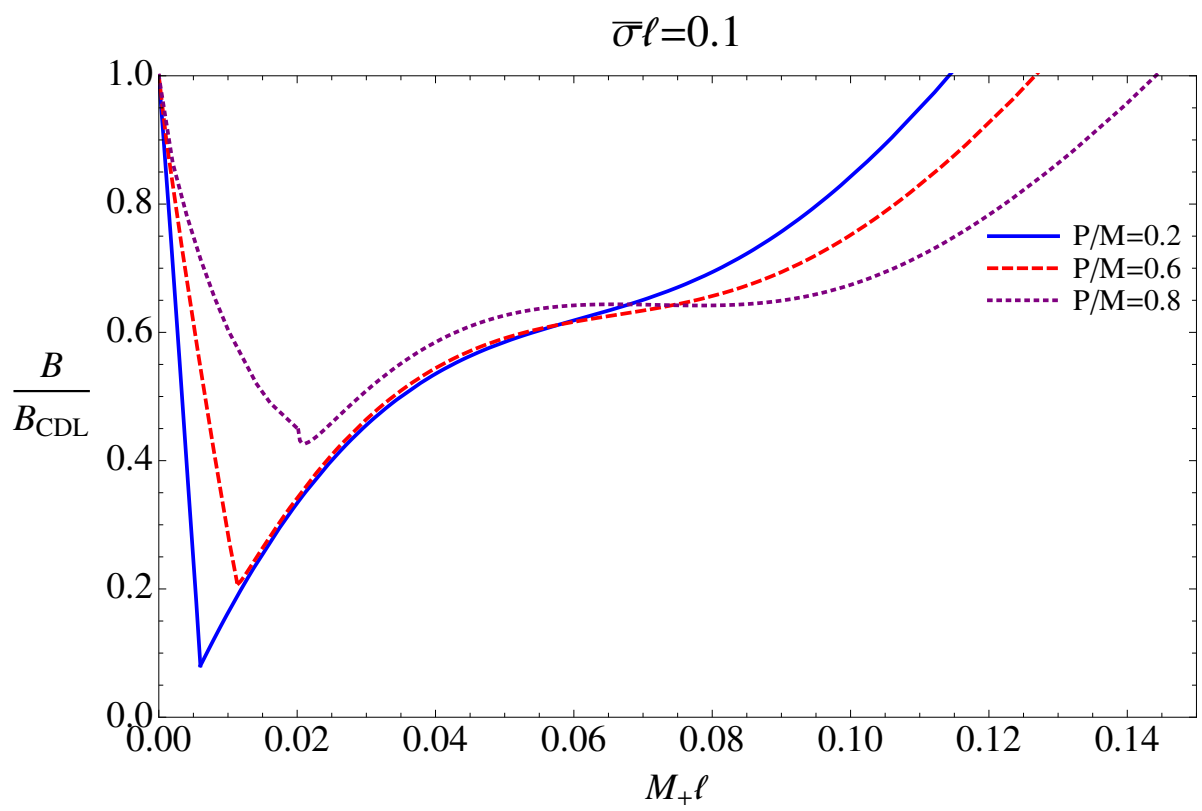

Figure 5. The exponent $B$ for the dominant tunnelling process with black-hole monopoles of mass $M_{+}$acting as nucleation seeds.

horizon area kicks in causing the usual rising of the bounce action. This small dip in the action near the critical point is very hard to see at low $P / M_{+}$, but for larger ratios becomes more visible. The dip is however very minor, and the minimum action is well approximated by the value at $M_{C P}$.

From figure 5 we see the dip is most visible at large ratio $P / M$, however, perhaps surprisingly, it is also the case that at large $P / M$ the catalytic effect of the black hole is much reduced. We therefore expect that the addition of a monopole charge will not particularly assist with vacuum decay, a conclusion largely borne out by the more detailed analysis of the next section.

\section{Application to the Higgs vacuum.}

Up to this point, the vacuum decay process has been described in gravitational terms using the surface tension of the wall, $\sigma$, and the AdS radius of the 'true' vacuum, $\ell$. In this section we will explore vacuum decay in the Higgs model with high energy corrections as discussed in the introduction. The key features of the potential relevant for quantum tunnelling are the barrier height, the separation between the minima and the energy of the true vacuum (TV). These three parameters can be encoded as follows,

$$
g=\phi_{T V} / M_{p}, \quad \epsilon=-V\left(\phi_{T V}\right), \quad \zeta=\sup _{0<\phi<\phi_{T V}} V(\phi)
$$

Following our previous discussion we shall restrict attention to potentials which allow thin-wall bubbles. Although we would expect $\zeta \gg \epsilon$ for a thin wall bubble, numerical solutions show that the wall approximation is reasonably accurate even when $\zeta \sim \epsilon$, therefore 


\begin{tabular}{|cccccccc|}
\hline$\lambda_{*}$ & $\phi_{*} / M_{p}$ & $\lambda_{6}$ & $g$ & $\ell / L_{p}$ & $\bar{\sigma} \ell$ & $M_{C} / M_{p}$ & $B_{C} / B_{C D L}$ \\
\hline-0.005 & 1 & 500 & 0.00146 & $3.17 \mathrm{e}+8$ & 0.00045 & 3.5 & $1.2 \mathrm{e}-6$ \\
-0.005 & 0.5 & $2 \mathrm{e}+03$ & 0.00073 & $1.27 \mathrm{e}+9$ & 0.00023 & 1.8 & $2.9 \mathrm{e}-7$ \\
-0.007 & 2 & $1.98 \mathrm{e}+03$ & 0.0008 & $9.33 \mathrm{e}+8$ & 0.00024 & 1.5 & $3.2 \mathrm{e}-7$ \\
-0.007 & 1 & $7.93 \mathrm{e}+03$ & 0.0004 & $3.79 \mathrm{e}+9$ & 0.00012 & 0.8 & $8.2 \mathrm{e}-8$ \\
-0.007 & 0.75 & $1.41 \mathrm{e}+04$ & 0.0003 & $6.76 \mathrm{e}+9$ & $9.1 \mathrm{e}-05$ & 0.61 & $4.7 \mathrm{e}-8$ \\
-0.007 & 0.5 & $3.17 \mathrm{e}+04$ & 0.0002 & $1.51 \mathrm{e}+10$ & $6 \mathrm{e}-05$ & 0.39 & $2 \mathrm{e}-8$ \\
-0.008 & 1 & $27 \mathrm{e}+03$ & 0.00022 & $1.18 \mathrm{e}+10$ & $6.9 \mathrm{e}-05$ & 0.46 & $2.7 \mathrm{e}-8$ \\
-0.008 & 3 & $3 \mathrm{e}+03$ & 0.00067 & $1.31 \mathrm{e}+9$ & 0.00021 & 1.4 & $2.4 \mathrm{e}-7$ \\
-0.009 & 1 & $85 \mathrm{e}+03$ & 0.00013 & $3.43 \mathrm{e}+10$ & $4.1 \mathrm{e}-05$ & 0.28 & $9.4 \mathrm{e}-9$ \\
-0.01 & 2 & $63 \mathrm{e}+03$ & 0.00016 & $2.55 \mathrm{e}+10$ & $5.4 \mathrm{e}-05$ & 0.49 & $1.7 \mathrm{e}-8$ \\
\hline
\end{tabular}

Table 1. A selection of parameter values for the modified Higgs potential, including the AdS radius $\ell$, the rescaled surface tension $\bar{\sigma} \ell$ and the critical mass $M_{C}$ for optimal nucleation seeded by a black hole. These parameters lie along the bottom edge of the parameter ranges for thin-wall bubbles. The vacuum tunnelling exponent $B_{C D L}$ is around $4 \mathrm{e}+06$ in each of these examples.

we use this lower bound for $\zeta$. The range of Higgs model parameters $\lambda_{*}, \phi_{*}$ and $\lambda_{6}$ which allow thin-wall bubbles is set by $\zeta>\epsilon>0$, and by the condition that the true vacuum lies at large $\phi$. Thin wall bubbles correspond to rather large values of $\lambda_{6}$, as illustrated in table 1. Roughly speaking, as $\lambda_{*}$ becomes more negative, the values of $\lambda_{6}$ required for thin-wall become larger, similarly as $\phi_{*}$ drops, $\lambda_{6}$ increases. In all cases the pure CDL tunneling action is extremely large $\left(10^{6-7}\right)$, but the suppression of the critical tunneling action is also large, and increases as $\lambda_{*}$ becomes more negative.

Following Coleman and De Luccia, we can express the surface tension of the bubble wall in terms of the potential. In order to extend the result to moderate values of $\zeta / \epsilon$, we compute the tension using the integral

$$
\sigma=\int_{0}^{\phi_{1}} d \phi(2 V)^{1 / 2} \simeq \kappa g M_{p} \zeta^{1 / 2},
$$

where the upper limit of the integral is at $V\left(\phi_{1}\right)=0$. The constant $\kappa$ depends on the details of the potential, but since $\phi_{1}<g M_{p}$ and $V \leq \xi$, it is subject to the constraint $\kappa<\sqrt{2}$. The AdS radius $\ell$ is related to the vacuum energy density by

$$
\ell=\sqrt{3} M_{p} \epsilon^{-1 / 2} .
$$

The back-reaction parameter $\bar{\sigma} \ell$ is therefore

$$
\bar{\sigma} \ell=\frac{1}{4 M_{p}^{2}} \sigma \ell=\frac{\sqrt{3}}{4} \kappa g\left(\frac{\zeta}{\epsilon}\right)^{1 / 2} .
$$


Note that $\bar{\sigma} \ell<1 / 2$ puts an upper bound on $g$. The CDL tunnelling exponent $B_{C D L}$ given in $(3.2)$ is

$$
B_{C D L}=\frac{27 \pi^{2} \sigma^{4}}{2 \epsilon^{3}\left(1-4 \bar{\sigma}^{2} \ell^{2}\right)^{2}}=\frac{27 \kappa^{4} \pi^{2}}{2}\left(\frac{g^{4} M_{p}^{4}}{\epsilon}\right)\left(\frac{\zeta}{\epsilon}\right)^{2}\left(1-4 \bar{\sigma}^{2} \ell^{2}\right)^{-2} .
$$

The large size of $B_{\mathrm{CDL}}$ in the parameter range covered by table 1 guarantees a tunnelling lifetime longer than the age of the universe (for unseeded nucleation).

When the vacuum decay is seeded by a black hole, the most rapid decay process occurs for a seed mass $M_{+}=M_{C}$ given in (3.4),

$$
M_{C} \approx \frac{128}{27} \frac{\ell}{G}(\bar{\sigma} \ell)^{3}=\frac{16}{3} \pi \kappa^{3}\left(\frac{g^{4} M_{p}^{4}}{\epsilon}\right)^{1 / 2}\left(\frac{\zeta}{\epsilon}\right)^{3 / 2} M_{p}
$$

The corresponding exponent in the nucleation rate is $B_{C}=0.5\left(M_{C} / M_{p}\right)^{2}$. Some values for $M_{C}$ are shown in table 1. If $M_{C} \gg M_{p}$, then the exponent $B_{C}$ is large and the seeded decay rate becomes vanishingly small. On the other hand, if $M_{C} \lesssim M_{p}$ then even if we have a seed mass $M_{+} \gg M_{C}$, we can still get significant suppression of the bounce action while remaining well above the Planck mass from tunneling on the static branch, as we see from figure 3. Our strategy therefore is to explore the decay seeded by a small mass black hole via the static instanton, which (for convenience) we determine numerically.

A brief consideration of the dependence of the bounce action on $M_{+}$shows that we are exploring seeded tunnelling for very light or primordial black holes [54], with temperatures well above that of the CMB. We must therefore check that the black hole can seed the false vacuum decay before it disappears through Hawking radiation. The vacuum decay rate $\Gamma_{D},(1.1)$, contains not only the exponential of the bounce action, but also a prefactor, A. According to Callan and Coleman [18], this pre-factor is made up of a factor of $(B / 2 \pi)^{1 / 2}$ for each translational zero mode of the instanton and a determinant factor. In our case, there will be a single zero mode representing the time translation symmetry, and rather than evaluate the determinant factor, we use the inverse horizon timescale as a rough estimate $\left(G M_{+}\right)^{-1}$, giving

$$
\Gamma_{D} \approx \sqrt{\frac{B}{2 \pi}} \frac{e^{-B}}{G M_{+}}
$$

The black hole emits Hawking radiation at a rate depending on fundamental particle masses and spins. The total decay rate for a subset of the standard model was evaluated by Page, [55]. If we set $\Gamma_{H}=\dot{M} / M$, then

$$
\Gamma_{H} \approx 3.6 \times 10^{-4}\left(G^{2} M_{+}^{3}\right)^{-1}
$$

The branching ratio of the tunnelling rate to the evaporation rate for uncharged black holes is therefore

$$
\frac{\Gamma_{D}}{\Gamma_{H}} \approx 44 \frac{M_{+}^{2}}{M_{p}^{2}} B^{1 / 2} e^{-B}
$$



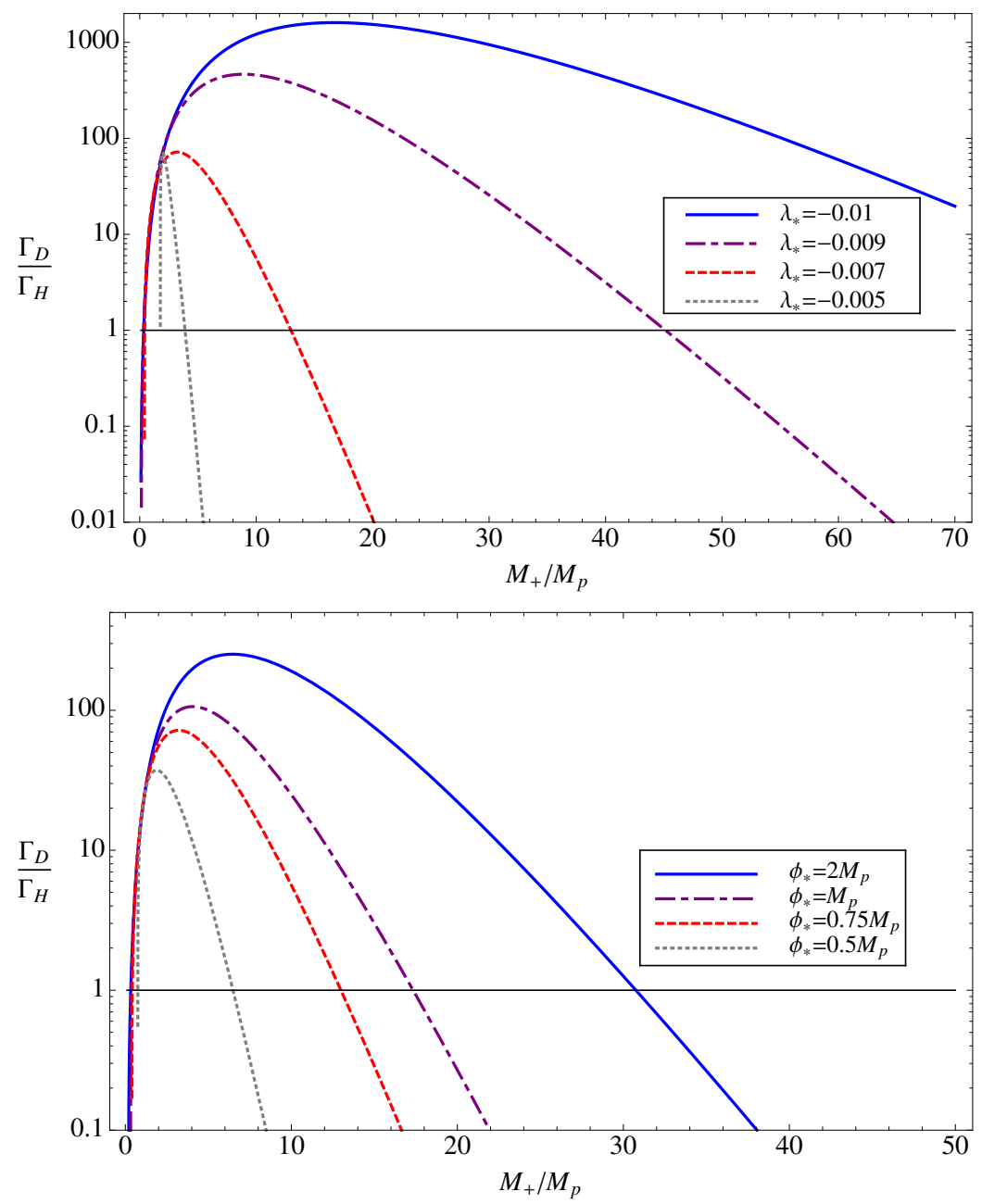

Figure 6. The branching ratio of the false vacuum nucleation rate to the Hawking evaporation rate as a function of the seed mass for a selection of Higgs models from table 1. The first plot shows the branching ratio for $\phi_{*}=M_{p}$ with the labelled values of $\lambda_{*}$, and the second for $\lambda_{*}=-0.007$ for the labelled values of $\phi_{*}$. The black hole starts out with a mass beyond the right-hand side of the plot and the mass decreases by Hawking evaporation. At some point, the vacuum decay rate becomes larger than the Hawking evaporation rate.

From this expression we can see roughly how the branching ratio will depend on $M_{+}$, even though $B$ is, in principle, a complex function of $M_{+}$. The static bubble action is the difference in areas of the seed and remnant black holes, which we can deduce from figure 4 to be roughly linear (there is actually a slightly stronger dependence on $M_{+}$, however, what is important is that it is not quadratic), whereas the prefactor is (again, roughly) $M_{+}^{5 / 2}$; we therefore expect the plot to be strongly exponentially suppressed at large $M_{+}$, but rising as $M_{+}$falls to a maximum around $M_{+} / M_{p}=\mathcal{O}(1)$, then dropping again below $M_{p}$. The actual value of the maximum will depend on the details of how $B$ depends on $M_{+}$, which requires a full calculation.

The branching ratio is plotted as a function of the seed mass $M_{+}$for two indicative sets of parameters taken from table 1 in figure 6 in order to illustrate the dependence on 


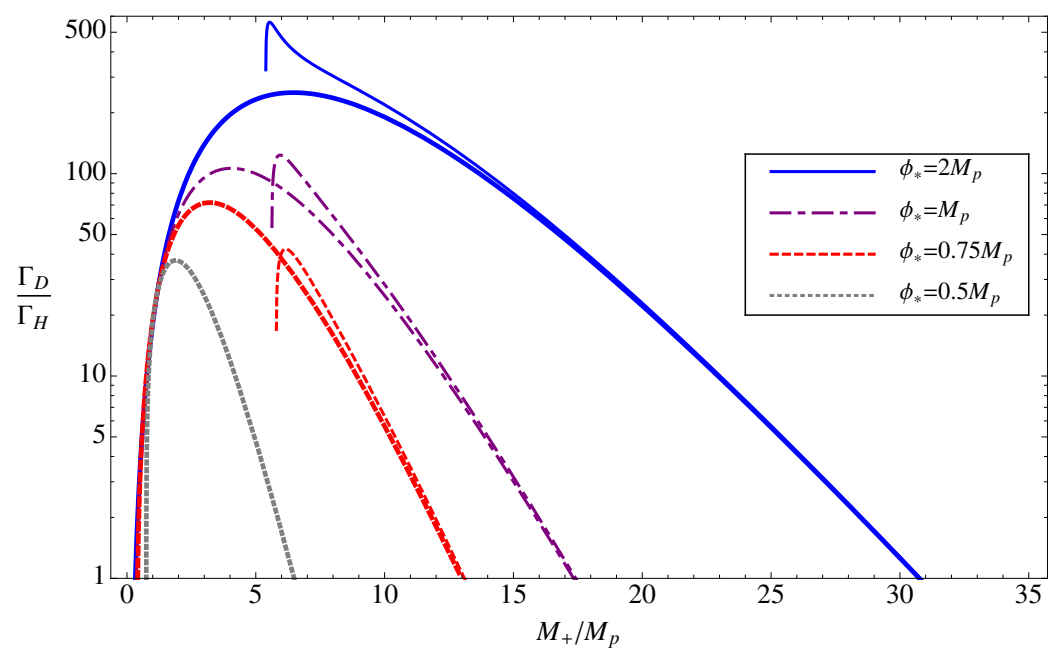

Figure 7. The branching ratio of the false vacuum nucleation rate to the Hawking evaporation rate for a monopole charged black hole with $P=5 M_{p}$, shown as a function of the seed mass for a selection of Higgs models from table 1. The plot for the uncharged black hole $(P=0)$ is repeated for comparison. As before, $\lambda_{*}=-0.007$ for the labelled values of $\phi_{*}$.

the parameters in the Higgs potential (or correspondingly on $\bar{\sigma}$ and $\ell$ ). The branching ratio is shown at fixed $\phi_{*}$ with varying $\lambda_{*}$ and vice versa. The overall picture is that for lower $\bar{\sigma}$ and higher $\ell$ (or more negative $\lambda_{*} /$ higher $\phi_{*}$ ) the branching ratio is larger, and is consistently higher than unity over a larger range. While Hawking evaporation always dominates at large $M_{+}$, the effect of Hawking radiation is that the black hole loses mass, hence driving it towards increasing branching ratio. A black hole produced in the early universe, for example, starts out with a mass well beyond the right-hand side of the plots, but at some point after evaporation, the vacuum decay rate becomes larger than the Hawking evaporation rate and the black hole seeds the transition to a new vacuum. This can occur for seed masses well above the Planck mass, where we have some confidence in the validity of the vacuum decay calculation. The timescales for Hawking evaporation and vacuum decay will both be less than roughly a million Planck times.

Finally, for the case of a monopole charged black hole, we might expect the branching ratio to be larger due to their reduced Hawking radiation rate: the Hawking flux is proportional to $\mathcal{A}_{+} T_{+}^{4}$, where $\mathcal{A}_{+}$is the event horizon, and $T_{+}$is the Hawking temperature

$$
T_{+}=\frac{1}{8 \pi G M_{+}} \frac{4 \Delta}{(1+\Delta)^{2}},
$$

setting $\Delta^{2}=1-P^{2} / M_{+}^{2}$. The evaporation rate is now

$$
\Gamma_{H} \approx 3.6 \times 10^{-4}\left(G^{2} M_{+}^{3}\right)^{-1} \frac{64 \Delta^{4}}{(1+\Delta)^{6}}
$$

The branching ratio $\Gamma_{D} / \Gamma_{H}$ can now be re-computed using this evaporation rate and the vacuum decay rates from the previous section. The result is shown in figure 7 . 


\section{Conclusions}

The main aim of this paper was to demonstrate that black holes can massively speed up the rate of decay of a metastable Higgs vacuum by acting as nucleation sites. We have shown that is the case, subject to the limitations of the analysis, some of which we shall now address.

We have used a Higgs model which was based loosely on the two-loop effective potential, with an extra term motivated by high-energy physics, possibly quantum gravity. This has enabled us to simplify the analysis by employing a thin-wall approximation, which is valid in part of the parameter space. The thin-wall approximation shows that when the seed mass is above some (calculable) critical mass, then the seeded nucleation proceeds via a static bubble solution. This gives a good starting point for an analysis of the thick wall bubble nucleation, which is far simpler for static than for non-static field configurations. We will show in a companion paper that the thick-wall bubble solutions extend the allowed range of parameters to small values of our new coefficient $\lambda_{6}$, which was rather large here, to the limit where $\lambda_{6}=0$, and the potential becomes simply the two-loop Higgs potential from the standard model.

The seeded nucleation calculation presented here requires a black hole, and for large tunneling enhancement, this is expected to be a primordial black hole which is evaporating and nearing the end of its life. There is of course another situation in which small black hole might occur. A possible alternative solution to the hierarchy problem has been to consider large extra dimensions, [56-59]. In these models, our universe is presumed to be a "brane" living within higher dimensions on which standard model physics is confined. The higher dimensional Planck scale is not hierarchically large, but instead our 4D Planck scale, which is derived via an integration over these extra dimensions, gains its leverage via the "large" internal volume. In such scenarios black holes can be created in particle collisions $[60,61]$, leading to considerable interest in the possibility of black holes being produced at the LHC (for a recent review see [62]). There are no known exact solutions for these black hole plus brane systems, and instead the black hole is usually considered to be approximately a higher dimensional Schwarzschild or Myers-Perry [63] solution (see [64, 65] for reviews on the issues and properties of brane black holes). For the Randall-Sundrum braneworld models, where the extra dimension is strongly warped, one could also consider "brane only" solutions, such as the tidal black hole [66].

Calculating the vacuum decay rates for these systems would be challenging, to say the least, not only because of the lack of a true higher dimensional black hole solution, but also because an instanton presumably would have to have a different vacuum only on the brane, and not in the bulk (although the braneworld equivalent of the CDL instantons were constructed in $[46,68]$ ). However, some features of our calculation should be present. The tidal black holes, for example, resemble black-hole monopoles, but with negative square monopole charge $P^{2}$. The bubble solutions for tidal holes are simple generalisations of the ones we have already discussed.

More concretely, in analogy with the use of the Myers-Perry geometries to approximate the small collider black holes, we could consider a higher dimensional analogue of our 


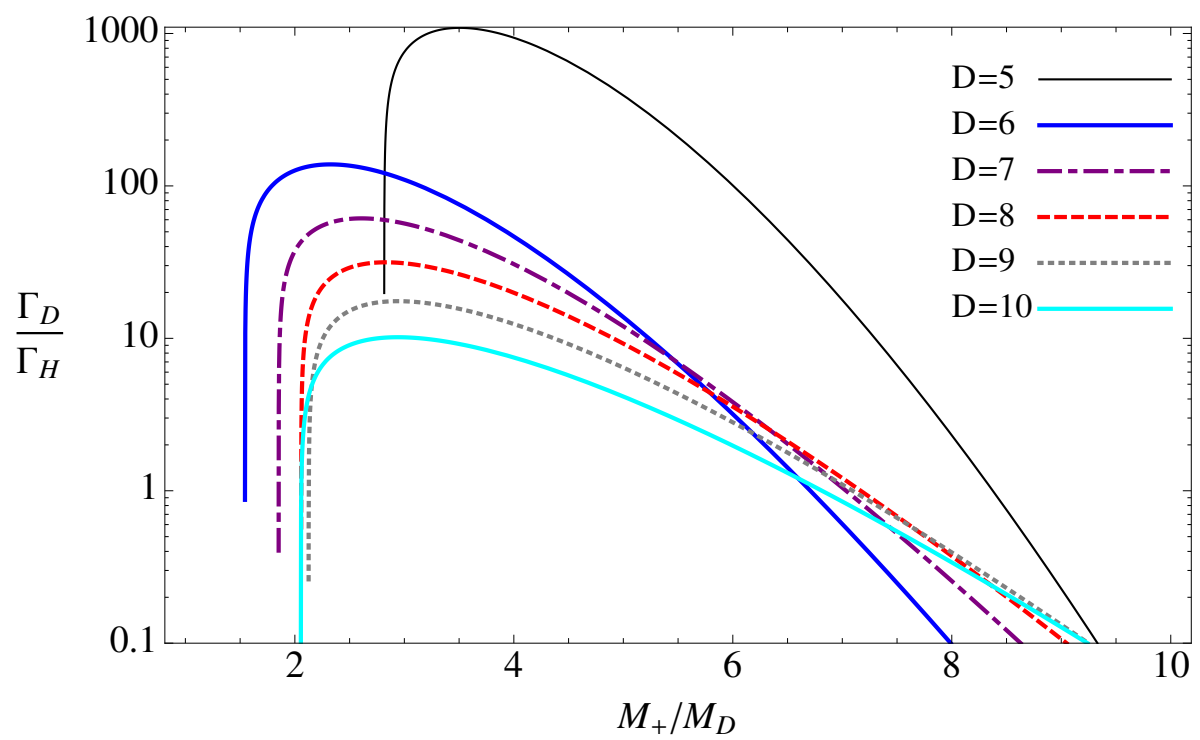

Figure 8. The dependence of the branching ratio on the dimensionality of spacetime, $D$. Here, the $D$-dimensional Planck mass is fixed, i.e. $8 \pi G_{D}$ always has the same value.

instanton. This would be a flawed model as it does not fit easily with a Higgs field which is confined to the brane, but it provides a concrete calculational method presumably as reasonable as the use of higher dimensional black hole geometries to approximate the brane black hole. Appendix $\mathrm{C}$ gives the details of the computation with the higher dimensional instanton, which leads to a dimension dependent branching ratio

$$
\frac{\Gamma_{D}}{\Gamma_{H}} \approx 550 \frac{M_{+} r_{+}}{(D-3)^{4}} \sqrt{B_{D}} e^{-B_{D}}=\frac{550 \sqrt{B_{D}} e^{-B_{D}}}{(D-3)^{4}}\left[\frac{16 \pi G_{D} M_{+}^{D-2}}{(D-2) A_{D-2}}\right]^{1 /(D-3)}
$$

where $B_{D}$ is given (implicitly) in (C.6). Defining the higher dimensional reduced Planck mass as ${ }^{1}$

$$
M_{D}^{D-2}=\frac{1}{8 \pi G_{D}}
$$

we can track the branching ratio as a function of $M_{+} / M_{D}$ and its dependence on $D$. To illustrate this dimensional dependence, we chose test-case values of $\bar{\sigma} \ell=0.01$ and $\ell=0.1$, and plotted $\Gamma_{D} / \Gamma_{H}$ in figure 8 .

The figure shows that as the number of extra dimensions increases, the branching ratio decreases, however the exact normalisation of the plot will depend on the confidence of translating modifications of the Higgs potential to the new Planck scale and variables. Given the crudeness of this particular model, we leave this, and possible refinements of the decay modelling to future work.

In conclusion, we have shown that the lifetime of our universe in a metastable Higgs phase is crucially dependent on the absence of any nucleation seeds, and a primordial black

\footnotetext{
${ }^{1}$ Note that in the literature, see e.g. [64], the non-reduced Planck mass is often used. Due to the dimension dependence of the Planck mass this will introduce various dimension dependent renormalisation factors between our expressions and those assumed there. Although these are of order unity, they do have some impact.
} 
hole could drastically reduce the time it takes to decay onto a different 'standard model'. Instability of the standard model is therefore more problematic than was hitherto supposed. Further exploration of the parameter space, using a wider class of bubble nucleation scenarios, should give us the range of Higgs parameters which lead to a long-lived standard model in the presence of black holes.

\section{Acknowledgments}

We would like to thank Ben Withers for collaboration in the early stages of this project, and also Niayesh Afshordi for useful discussions.

PB is supported by an EPSRC International Doctoral Scholarship, RG and IGM are supported in part by STFC (Consolidated Grant ST/J000426/1). RG is also supported by the Wolfson Foundation and Royal Society, and Perimeter Institute for Theoretical Physics. Research at Perimeter Institute is supported by the Government of Canada through Industry Canada and by the Province of Ontario through the Ministry of Research and Innovation.

\section{A The limits on $k_{1}, k_{2}$}

The values of $k_{1}$ and $k_{2}$ are limited by requiring existence of a solution to $\dot{\tilde{R}}^{2}+U(\tilde{R})=0$, where $U$ is defined in (2.10), requiring positivity of the black hole masses, and positivity of the arrows of time on each side of the wall:

$$
\begin{aligned}
& f_{+} \frac{d \tilde{\tau}_{+}}{d \tilde{\lambda}}=\frac{k_{2}}{\tilde{R}^{2}}+\frac{\tilde{R}}{\alpha}(1-2 \bar{\sigma} \gamma) \\
& f_{-} \frac{d \tilde{\tau}_{-}}{d \tilde{\lambda}}=\frac{k_{2}}{\tilde{R}^{2}}+\frac{\tilde{R}}{\alpha}
\end{aligned}
$$

where

$$
\begin{aligned}
& f_{+}=1-\frac{k_{1}}{\tilde{R}}-\frac{2 k_{2}}{\alpha \tilde{R}}(\alpha-(1-2 \bar{\sigma} \gamma))-\frac{\tilde{R}^{2}}{\alpha^{2}}\left[\alpha^{2}-(1-2 \bar{\sigma} \gamma)^{2}\right] \\
& f_{-}=1-\frac{k_{1}}{\tilde{R}}+\frac{2 k_{2}}{\alpha \tilde{R}}(1-\alpha)+\frac{\tilde{R}^{2}}{\alpha^{2}}\left(1-\alpha^{2}\right) .
\end{aligned}
$$

The first requirement is algebraically identical to the constraint discussed in [21] (although the expression given there in the appendix was not correct). Simultaneously requiring $U=U^{\prime}=0$ and eliminating $\tilde{R}_{*}$ gives an upper limit $k_{1} \leq k_{1}^{*}$, the correct expression for which is:

$$
\begin{aligned}
k_{1}^{*}=\frac{2}{9} & {\left[1+81 k_{2}^{2}-\left(-1-5\left(27 k_{2}\right)^{2}+\frac{\left(27 k_{2}\right)^{4}}{2}+\frac{27 k_{2}}{2}\left(4+\left(27 k_{2}\right)^{2}\right)^{3 / 2}\right)^{1 / 3}\right.} \\
& \left.+\left(1+5\left(27 k_{2}\right)^{2}-\frac{\left(27 k_{2}\right)^{4}}{2}+\frac{27 k_{2}}{2}\left(4+\left(27 k_{2}\right)^{2}\right)^{3 / 2}\right)^{1 / 3}\right]^{1 / 2}-2 k_{2}
\end{aligned}
$$

To get lower limits for $k_{1}$ we have to consider positivity of the black hole masses and the arrows of time on each side of the wall. These now depend on the sign and magnitude 
of the cosmological constants and are different to [21]. First, note the relation between the physical quantities and the parameters:

$$
\begin{aligned}
G M_{-} & =\frac{\gamma}{2 \alpha}\left(k_{1}-2 \frac{(1-\alpha)}{\alpha} k_{2}\right) \\
G M_{+} & =\frac{\gamma k_{1}}{2 \alpha}+\frac{k_{2} \gamma}{\alpha^{2}}(\alpha-1+2 \bar{\sigma} \gamma) \\
\Lambda_{-} & =3 \frac{\alpha^{2}-1}{\gamma^{2}} \\
\Lambda_{+} & =\Lambda_{-}-12 \bar{\sigma}^{2}+12 \frac{\bar{\sigma}}{\gamma}=\frac{3}{\gamma^{2}}\left(\alpha^{2}-(1-2 \bar{\sigma} \gamma)^{2}\right)
\end{aligned}
$$

thus

$$
\begin{aligned}
& G M_{-} \geq 0 \quad \Rightarrow \quad k_{1} \geq 2(1-\alpha) \frac{k_{2}}{\alpha} \\
& G M_{+} \geq 0 \quad \Rightarrow \quad k_{1} \geq 2(1-\alpha+2 \bar{\sigma} \gamma) \frac{k_{2}}{\alpha}
\end{aligned}
$$

Secondly, the requirement of positivity of $\dot{\tilde{\tau}}_{ \pm}$, of which the constraint on $\dot{\tilde{\tau}}_{+}$is the stronger:

$$
\frac{k_{2}}{\tilde{R}^{2}}+\frac{\tilde{R}}{\alpha}(1-2 \bar{\sigma} \gamma) \geq 0
$$

saturated by $\tilde{R}_{+}^{3}=\alpha k_{2} /(2 \bar{\sigma} \gamma-1)$. Clearly, if $2 \bar{\sigma} \gamma>1$, we must have $k_{2}>0$, and $U$ must be positive with positive gradient at $\tilde{R}_{+}$. A brief manipulation of $U^{\prime}>0$ yields

$$
\frac{\alpha^{2}}{(2 \bar{\sigma} \gamma-1)^{2}}-2-\frac{k_{1}+2 k_{2}}{2 k_{2}} \frac{\alpha}{(2 \bar{\sigma} \gamma-1)} \geq 0
$$

From this, we see that $\Lambda_{+}>0,2 \bar{\sigma} \gamma \leq 1+\alpha / 2$, and $k_{1}$ is bounded below by $k_{1}^{m}=$ $2(1-\alpha) k_{2} / \alpha$ from (A.6).

Now consider $2 \bar{\sigma} \gamma<1 / 2$, for which $k_{2}<0$ is allowed. A similar argument to [21] gives

$$
k_{1} \geq k_{1}^{m}=\left|\frac{\alpha k_{2}}{2 \bar{\sigma} \gamma-1}\right|^{1 / 3}+\frac{k_{2}}{(1-2 \bar{\sigma} \gamma)} \frac{(\alpha-1+2 \bar{\sigma} \gamma)^{2}}{\alpha}
$$

for $k_{2} \leq 0$, and once again, $k_{1}^{m}=2(1-\alpha) k_{2} / \alpha$ for $k_{2}>0$. Note that neither of these bounds requires a particular sign for $\Lambda_{-}$.

Where the sign of $\Lambda_{-}$does make a difference is in the range of allowed $k_{2}$. The upper limit on $k_{2}$ is determined by $M_{-}=0$ for the static bounce, i.e.

$$
\alpha k_{1}^{*}\left[k_{2}\right]=2(1-\alpha) k_{2}
$$

and a lower bound on $k_{2}$ is determined when the range of $k_{1}$ for $k_{2}<0$ closes off at negative $k_{2}$, which occurs when $U\left(\tilde{R}_{+}\right)=U^{\prime}\left(\tilde{R}_{+}\right)=0$ :

$$
\tilde{R}_{+}^{4}-3 \tilde{R}_{+}^{6}+3 k_{2}^{2}=\tilde{R}_{+}^{4}-\frac{\gamma^{2} k_{2}^{2}}{(1-2 \bar{\sigma} \gamma)^{2}} \Lambda_{+}=0
$$




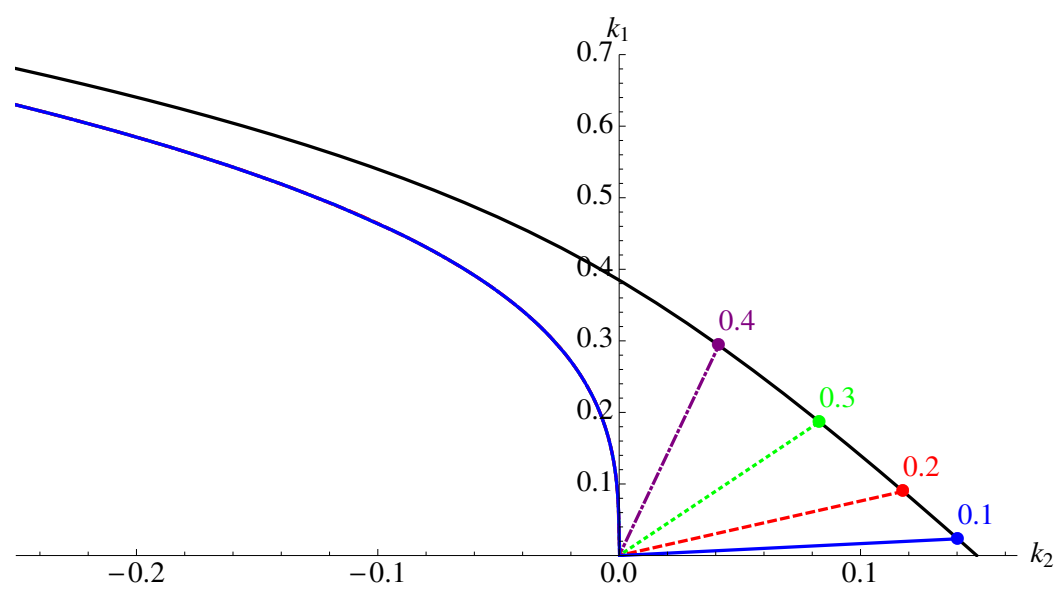

Figure 9. The allowed ranges for the parameters $k_{1}$ and $k_{2}$ with $\Lambda_{+}=0$. The upper bound on $k_{1}$ corresponds to stationary wall solutions. The lower bound on $k_{1}$ when $k_{2}>0$ corresponds to vanishing remnant mass $M_{-}=0$. The two limits intersect at a point $\left(k_{1 C}, k_{2 C}\right)$, which depends on the $\bar{\sigma} \ell$ and approaches $(0,4 / 27)$ as $\bar{\sigma} \ell \rightarrow 0$. The strip of allowed $k_{1}$ continues to the left as $\left|k_{2}\right|^{1 / 3}$.

which is clearly inconsistent for $\Lambda_{+} \leq 0$. For $\Lambda_{+}>0$, the range closes off at

$$
k_{2, \min }=\frac{\alpha^{2}(2 \bar{\sigma} \gamma-1)}{3 \sqrt{3}\left[\alpha^{2}-(1-2 \bar{\sigma} \gamma)^{2}\right]^{3 / 2}}=\frac{\alpha^{2}(2 \bar{\sigma} \gamma-1)}{\gamma^{3} \Lambda_{+}^{3 / 2}}
$$

Note that for $\Lambda_{+}<0$, the range of $k_{1}$, while initially narrowing as $k_{2}$ becomes negative, eventually opens out, as the linear term in (A.9) becomes dominant. Thus large AdS black holes can tunnel to an even larger AdS black hole with a more negative cosmological constant.

In this paper, we mostly focus on $\Lambda_{+}=0$, for which $\alpha=1-2 \bar{\sigma} \gamma$, and many of the expressions above simplify:

$$
k_{1}^{m}= \begin{cases}\left(-k_{2}\right)^{1 / 3} & k_{2} \leq 0 \\ \frac{2(1-\alpha)}{\alpha} k_{2} & k_{2}>0\end{cases}
$$

and the range of $k_{2}$ is plotted in figure 9. Note that unlike the figure in [21], where the lower limit for $k_{1}$ was dependent on $\bar{\sigma}$ for negative but not positive $k_{2}$, in this case it is the lower limits for positive $k_{2}$ and not negative that depend on $\bar{\sigma}$.

\section{B Alternative bounce action calculation}

In this appendix we present an alternative derivation of the general expression for the bounce action using the Hamiltonian approach presented in [21], and extend the result in some cases beyond the thin-wall limit. We will evaluate the Euclidean action for gravity plus a scalar field, with Lagrangian $\mathcal{L}_{m}$, in an asymptotically AdS or flat spacetime. As in $\S 2.2$, we take the action on a sequence $\mathcal{M}_{r}$ of manifolds with boundary $\partial \mathcal{M}_{r}$ at $r_{0}$, and 
subtract the action of a similar sequence $\overline{\mathcal{M}}_{r}$ of Euclidean AdS manifolds with the same boundary $\partial \mathcal{M}_{r}$.

It is instructive to first consider the case where the Euclidean spacetime $\mathcal{M}$ is perfectly regular, with no conical singularities, and has a Killing vector, $\partial_{\tau}$. We perform a foliation of $\mathcal{M}_{r}$ with a family of non-intersecting surfaces $\Sigma_{\tau}$ (assuming the global topology permits), with $0<\tau<\beta$. The canonical decomposition of such foliations has been investigated by Hawking and Horowitz, [67]. In order to decompose the action, we use their identity

$$
\mathcal{R}={ }^{3} \mathcal{R}-K^{2}+K_{a b}^{2}-2 \nabla_{a}\left(u^{a} \nabla_{b} u^{b}\right)+2 \nabla_{b}\left(u^{a} \nabla_{a} u^{b}\right),
$$

where the vector $u^{\mu}$ is normal to $\Sigma_{\tau}$ and ${ }^{3} \mathcal{R}$ is the Ricci-curvature of $\Sigma_{\tau}$. The action therefore splits into bulk and boundary parts,

$$
\begin{aligned}
I_{\mathcal{M}_{r}}=- & \frac{1}{16 \pi G} \int_{0}^{\beta} d \tau \int_{\Sigma_{\tau}}\left({ }^{3} \mathcal{R}-K^{2}+K_{a b}^{2}+16 \pi G \mathcal{L}_{m}\right) \sqrt{g} \\
& +\frac{1}{8 \pi G} \int_{\partial \mathcal{M}_{r}} n_{b} u^{a} \nabla_{a} u^{b} \sqrt{h} .
\end{aligned}
$$

The bulk term expressed in terms of canonical momenta $\pi^{i j}$ and $\pi$ becomes

$$
\frac{1}{16 \pi G} \int_{0}^{\beta} d \tau\left[\int_{\Sigma_{\tau}}\left(\partial_{\tau} \gamma_{i j} \pi^{i j}+\partial_{\tau} \phi \pi-N \mathcal{H}-N^{i} \mathcal{H}_{i}\right) \sqrt{h}\right],
$$

where $\mathcal{H}$ and $\mathcal{H}_{i}$ are the Hamiltonian and momentum constraints respectively. The field equations imply that $\mathcal{H}=\mathcal{H}_{i}=0$, and furthermore the symmetry implies $\partial_{\tau} \phi=\partial_{\tau} \gamma_{i j}=0$. Therefore only the boundary term in (B.2) survives.

To evaluate the boundary term, we use the fact that the metric is static and asymptotically AdS, therefore at large $r$ we have

$$
d s^{2}=f(r) d \tau^{2}+f(r)^{-1} d r^{2}+r^{2} d \Omega^{2}
$$

where $f=1-2 G M / r-\Lambda r^{2} / 3$. For this metric $n_{b} u^{a} \nabla_{a} u^{b}=f^{-1 / 2} f^{\prime} / 2$, and subtracting the Euclidean AdS action from (B.2) we arrive at the following expression

$$
I=\lim _{r \rightarrow \infty}\left(\frac{\beta r^{2} f^{\prime}}{4 G}-\frac{\beta_{0} r^{2} f_{0}^{\prime}}{4 G}\right)
$$

where $\beta_{0}$ and $f_{0}^{\prime}$ are the time-period and metric function of AdS space, and using (2.17) the Euclidean action (B.5) becomes,

$$
I=\beta M
$$

If there is a horizon at $r=r_{h}$, then by properly treating the conical singularity as in [21], we get an additional area term contribution to the action

$$
I=\beta M-\frac{1}{4 G} \mathcal{A}_{h} .
$$

This result generalises a previous result of Hawking and Horowitz, who found the same formula for the Euclidean action of static Einstein-matter solutions with $\Lambda=0$ and no 
conical singularities [67]. The $\Lambda \rightarrow 0$ case can also be obtained using the Gibbons-Hawking subtraction procedure described in $\S 2.2$. The expression (B.7) is of course the same as (2.16), (2.19), but we have not assumed anywhere for the static case that the bubble is in the thin-wall limit.

Solutions with a moving bubble wall $(\tau(\lambda), R(\lambda))$ break the time-translation symmetry of the full space-time, but the canonical method can still be used if the wall is thin and the geometries on both sides of the bubble wall, $\mathcal{M}_{ \pm}$, individually possess the Killing vector, $\partial_{\tau}$. Along with the contributions from $\mathcal{M}_{ \pm}$and the wall, $\mathcal{W}$, we will have an additional contribution from the conical singularity which can be dealt with by the methods of [21]. The action splits into contributions from each of these parts

$$
I_{\mathcal{M}_{r}}=I_{-}+I_{+}+I_{\mathcal{W}}-\frac{\mathcal{A}_{-}}{4 G}
$$

where $\mathcal{A}_{-}$is the area of the black hole horizon in the interior of the bubble. In the thin wall limit $I_{\mathcal{W}}=\int_{\mathcal{W}} \sigma$, and

$$
I_{ \pm}=-\frac{1}{16 \pi G} \int_{\mathcal{M}_{ \pm}} \mathcal{R} \sqrt{g}-\int_{\mathcal{M}_{ \pm}} \mathcal{L}_{m}(g, \phi) \sqrt{g}+\frac{1}{8 \pi G} \int_{\mathcal{W}} K_{ \pm} \sqrt{h},
$$

Hence, performing the same decomposition as in the static case we can cancel the bulk contributions and are left only with the boundary terms

$$
I_{ \pm}=-\frac{1}{8 \pi G} \int_{\mathcal{W}} K_{ \pm} \sqrt{h}+\frac{1}{8 \pi G} \int_{\partial \mathcal{M}_{ \pm}} n_{ \pm b} u^{a} \nabla_{a} u^{b} \sqrt{h},
$$

where $\partial \mathcal{M}_{+}$now includes the wall and the large distance boundary $\partial \mathcal{M}_{r}$. Using Israel's junction condition to relate the extrinsic curvatures on each side of the wall to the tension, and inserting the normal to the wall $(2.3), n_{b} u^{a} \nabla_{a} u^{b}=\dot{\tau} f^{-1 / 2} f^{\prime} / 2$, we reach our final result

$$
I=\beta M_{+}-\frac{1}{4 G} \mathcal{A}_{-}-\frac{1}{2} \int_{\mathcal{W}} \sigma \sqrt{h}-\frac{1}{16 \pi G} \int_{\mathcal{W}}\left(f_{+}^{\prime} \dot{\tau}_{+}-f_{-}^{\prime} \dot{\tau}_{-}\right) \sqrt{h}
$$

The expression for the bounce action $B$ which governs the decay rate is obtained from $I$ by subtracting the background action without the bubble, $I_{0}$ :

$$
I_{0}=\beta M_{+}-\frac{1}{4 G} \mathcal{A}_{+} .
$$

Therefore the tunnelling rate is determined by

$$
B=\frac{\mathcal{A}_{+}}{4 G}-\frac{\mathcal{A}_{-}}{4 G}-\frac{1}{2} \int_{\mathcal{W}} \sigma \sqrt{h}-\frac{1}{16 \pi G} \int_{\mathcal{W}}\left(f_{+}^{\prime} \dot{\tau}_{+}-f_{-}^{\prime} \dot{\tau}_{-}\right) \sqrt{h},
$$

which is identical to $(2.22)$, after using the relation $f_{+} \dot{\tau}_{+}-f_{-} \dot{\tau}_{-}=-2 \bar{\sigma} R$ for the wall integral.

\section{Higher dimensional instantons}

Here we briefly outline the simple higher dimensional instanton model. Note this is only a crude calculation meant to give an indication of the effect of large extra dimensions, and is 
problematic as an approximation to an actual Higgs tunneling event due to the lack of an exact braneworld black hole solution, as well as the fact that the Higgs should be confined to the brane, hence the instanton should instead involve only a shift in the cosmological constant on the brane. We take a higher dimensional black hole solution with different masses and cosmological constant on each side of the wall, and compute how the branching ratio changes with dimension.

The equations of motion have the same schematic form as (2.6)

$$
\dot{R}^{2}=\bar{\sigma}^{2} R^{2}-\bar{f}+\frac{(\Delta f)^{2}}{16 \bar{\sigma}^{2} R^{2}}
$$

but with $\bar{\sigma}=4 \pi G \sigma /(D-2)[46,68]$, and $f$ now the higher dimensional Schwarzschild potential:

$$
f=1-\frac{2 \Lambda r^{2}}{(D-1)(D-2)}-\frac{16 \pi G_{D} M}{(D-2) A_{D-2} r^{D-3}}
$$

where $G_{D}$ is the higher dimensional Newton constant and $A_{D-2}=2 \pi^{\frac{D+1}{2}} / \Gamma\left[\frac{D+1}{2}\right]$.

Defining $\ell, \gamma$ and $\alpha$ in a similar fashion:

$$
\ell^{2}=\frac{(D-1)(D-2)}{2 \Delta \Lambda} \quad, \quad \gamma=\frac{4 \bar{\sigma} \ell^{2}}{1+4 \bar{\sigma}^{2} \ell^{2}} \quad, \quad \alpha^{2}=1+\frac{2 \Lambda_{-} \gamma^{2}}{(D-1)(D-2)}
$$

and

$$
\begin{aligned}
& k_{1}=\frac{16 \pi G_{D}}{(D-2) A_{D-2}}\left(\frac{\alpha}{\gamma}\right)^{D-3}\left[M_{-}+(1-\alpha) \frac{\Delta M}{2 \bar{\sigma} \gamma}\right] \\
& k_{2}=\frac{16 \pi G_{D}}{(D-2) A_{D-2}}\left(\frac{\alpha}{\gamma}\right)^{D-2} \frac{\Delta M}{4 \bar{\sigma}}
\end{aligned}
$$

Then setting $\tilde{R}=\alpha R / \gamma, \tilde{\lambda}=\alpha \lambda / \gamma$ gives the equation of motion

$$
\left(\frac{d \tilde{R}}{d \tilde{\lambda}}\right)^{2}=1-\tilde{R}^{2}-\frac{k_{1}+2 k_{2}}{\tilde{R}^{D-3}}-\frac{k_{2}^{2}}{\tilde{R}^{2(D-2)}}
$$

which is of the same form as (2.10) albeit with different exponents of $\tilde{R}$. We can use the same procedure as before to find the static solution and the dynamical bubble which removes the black hole. The static action (which is what is needed for the branching ratio) is, as before, the difference in horizon areas:

$$
B_{D}=\frac{A_{D-2}}{4 G}\left(r_{+}^{D-2}-r_{-}^{D-2}\right)
$$

where $r_{ \pm}$are determined numerically, and the corresponding tunneling rate is

$$
\Gamma_{D}=\sqrt{\frac{B_{D}}{2 \pi}} \frac{e^{-B_{D}}}{r_{+}}
$$

Meanwhile the Hawking temperature of the higher dimensional black hole is [63, 64]

$$
T_{H}=\frac{(D-3)}{4 \pi r_{+}}
$$


To estimate the decay rate of the black hole, we will assume that the main channel is due to emission of particles on the brane $[69,70]$, leading to

$$
\Gamma_{H} \sim 3.6 \times 10^{-4} \frac{64 \pi G_{D}}{A_{D-2} r_{+}^{D-1}} \frac{(D-3)^{4}}{(D-2)}
$$

hence a branching ratio of

$$
\frac{\Gamma_{D}}{\Gamma_{H}} \approx 550 \frac{M_{+} r_{+}}{(D-3)^{4}} \sqrt{B_{*}} e^{-B_{*}}
$$

Open Access. This article is distributed under the terms of the Creative Commons Attribution License (CC-BY 4.0), which permits any use, distribution and reproduction in any medium, provided the original author(s) and source are credited.

\section{References}

[1] ATLAS collaboration, Combined search for the Standard Model Higgs boson using up to $4.9 \mathrm{fb}^{-1}$ of pp collision data at $\sqrt{s}=7 \mathrm{TeV}$ with the ATLAS detector at the LHC, Phys. Lett. B $\mathbf{7 1 0}$ (2012) 49 [arXiv: 1202.1408] [INSPIRE].

[2] CMS collaboration, Combined results of searches for the standard model Higgs boson in pp collisions at $\sqrt{s}=7 \mathrm{TeV}$, Phys. Lett. B 710 (2012) 26 [arXiv:1202.1488] [INSPIRE].

[3] G. Degrassi et al., Higgs mass and vacuum stability in the standard model at NNLO, JHEP 08 (2012) 098 [arXiv: 1205.6497] [INSPIRE].

[4] A. Gorsky, A. Mironov, A. Morozov and T.N. Tomaras, Is the standard model saved asymptotically by conformal symmetry?, J. Exp. Theor. Phys. 120 (2015) 344 [arXiv: 1409.0492] [INSPIRE].

[5] F. Bezrukov and M. Shaposhnikov, Why should we care about the top quark Yukawa coupling?, J. Exp. Theor. Phys. 120 (2015) 335 [arXiv:1411.1923] [INSPIRE].

[6] J. Ellis, Discrete glimpses of the physics landscape after the Higgs discovery, J. Phys. Conf. Ser. 631 (2015) 012001 [arXiv: 1501.05418] [INSPIRE].

[7] K. Blum, R.T. D'Agnolo and J. Fan, Vacuum stability bounds on Higgs coupling deviations in the absence of new bosons, JHEP 03 (2015) 166 [arXiv:1502.01045] [INSPIRE].

[8] M.E. Bracco, G. Krein and M. Nielsen, Quark meson coupling model with constituent quarks: exchange and pionic effects, Phys. Lett. B 432 (1998) 258 [nucl-th/9805019] [INSPIRE].

[9] N. Cabibbo, L. Maiani, G. Parisi and R. Petronzio, Bounds on the fermions and Higgs boson masses in grand unified theories, Nucl. Phys. B 158 (1979) 295 [INSPIRE].

[10] M.S. Turner and F. Wilczek, Is our vacuum metastable, Nature D 79 (1982) 633.

[11] M. Lindner, M. Sher and H.W. Zaglauer, Probing vacuum stability bounds at the Fermilab collider, Phys. Lett. B 228 (1989) 139 [INSPIRE].

[12] M. Sher, Electroweak Higgs potentials and vacuum stability, Phys. Rept. 179 (1989) 273 [INSPIRE].

[13] G. Isidori, G. Ridolfi and A. Strumia, On the metastability of the standard model vacuum, Nucl. Phys. B 609 (2001) 387 [hep-ph/0104016] [INSPIRE]. 
[14] J.R. Espinosa, G.F. Giudice and A. Riotto, Cosmological implications of the Higgs mass measurement, JCAP 05 (2008) 002 [arXiv:0710.2484] [INSPIRE].

[15] G. Isidori, V.S. Rychkov, A. Strumia and N. Tetradis, Gravitational corrections to standard model vacuum decay, Phys. Rev. D 77 (2008) 025034 [arXiv:0712.0242] [INSPIRE].

[16] J. Elias-Miro, J.R. Espinosa, G.F. Giudice, G. Isidori, A. Riotto and A. Strumia, Higgs mass implications on the stability of the electroweak vacuum, Phys. Lett. B 709 (2012) 222 [arXiv:1112.3022] [INSPIRE].

[17] S.R. Coleman, The fate of the false vacuum. 1. Semiclassical theory, Phys. Rev. D 15 (1977) 2929 [Erratum ibid. D 16 (1977) 1248] [INSPIRE].

[18] C.G. Callan Jr. and S.R. Coleman, The fate of the false vacuum. 2. First quantum corrections, Phys. Rev. D 16 (1977) 1762 [INSPIRE].

[19] S.R. Coleman and F. De Luccia, Gravitational effects on and of vacuum decay, Phys. Rev. D 21 (1980) 3305 [INSPIRE].

[20] I. Yu. Kobzarev, L.B. Okun and M.B. Voloshin, Bubbles in metastable vacuum, Sov. J. Nucl. Phys. 20 (1975) 644 [INSPIRE].

[21] R. Gregory, I.G. Moss and B. Withers, Black holes as bubble nucleation sites, JHEP 03 (2014) 081 [arXiv:1401.0017] [INSPIRE].

[22] W.A. Hiscock, Can black holes nucleate vacuum phase transitions?, Phys. Rev. D 35 (1987) 1161 [INSPIRE].

[23] V.A. Berezin, V.A. Kuzmin and I.I. Tkachev, $O(3)$ invariant tunneling in general relativity, Phys. Lett. B 207 (1988) 397 [INSPIRE].

[24] M. Sasaki and D.-h. Yeom, Thin-shell bubbles and information loss problem in Anti de Sitter background, JHEP 12 (2014) 155 [arXiv:1404.1565] [INSPIRE].

[25] A. Shkerin and S. Sibiryakov, On stability of electroweak vacuum during inflation, Phys. Lett. B 746 (2015) 257 [arXiv: 1503.02586] [InSPIRE].

[26] P. Burda, R. Gregory and I. Moss, Gravity and the stability of the Higgs vacuum, Phys. Rev. Lett. 115 (2015) 071303 [arXiv: 1501.04937] [INSPIRE].

[27] F. Mellor and I. Moss, Black holes and quantum wormholes, Phys. Lett. B 222 (1989) 361 [INSPIRE].

[28] F. Mellor and I. Moss, Black holes and gravitational instantons, Class. Quant. Grav. 6 (1989) 1379 [INSPIRE].

[29] S.W. Hawking and N. Turok, Open inflation without false vacua, Phys. Lett. B 425 (1998) 25 [hep-th/9802030] [INSPIRE].

[30] N. Turok and S.W. Hawking, Open inflation, the four form and the cosmological constant, Phys. Lett. B 432 (1998) 271 [hep-th/9803156] [INSPIRE].

[31] A.R. Brown and E.J. Weinberg, Thermal derivation of the Coleman-De Luccia tunneling prescription, Phys. Rev. D 76 (2007) 064003 [arXiv:0706.1573] [InSPIRE].

[32] I.G. Moss, Black hole bubbles, Phys. Rev. D 32 (1985) 1333 [inSPIRE].

[33] C. Cheung and S. Leichenauer, Limits on new physics from black holes, Phys. Rev. D 89 (2014) 104035 [arXiv:1309.0530] [INSPIRE]. 
[34] C. Ford, D.R.T. Jones, P.W. Stephenson and M.B. Einhorn, The effective potential and the renormalization group, Nucl. Phys. B 395 (1993) 17 [hep-lat/9210033] [INSPIRE].

[35] K.G. Chetyrkin and M.F. Zoller, Three-loop $\beta$-functions for top-Yukawa and the Higgs self-interaction in the standard model, JHEP 06 (2012) 033 [arXiv:1205.2892] [INSPIRE].

[36] F. Bezrukov, M.Y. Kalmykov, B.A. Kniehl and M. Shaposhnikov, Higgs boson mass and new physics, JHEP 10 (2012) 140 [arXiv:1205.2893] [INSPIRE].

[37] B. Bergerhoff, M. Lindner and M. Weiser, Dynamics of metastable vacua in the early universe, Phys. Lett. B 469 (1999) 61 [hep-ph/9909261] [InSPIRE].

[38] E. Greenwood, E. Halstead, R. Poltis and D. Stojkovic, Dark energy, the electroweak vacua and collider phenomenology, Phys. Rev. D 79 (2009) 103003 [arXiv:0810.5343] [InSPIRE].

[39] V. Branchina and E. Messina, Stability, Higgs boson mass and new physics, Phys. Rev. Lett. 111 (2013) 241801 [arXiv:1307.5193] [INSPIRE].

[40] V. Branchina, E. Messina and M. Sher, Lifetime of the electroweak vacuum and sensitivity to Planck scale physics, Phys. Rev. D 91 (2015) 013003 [arXiv:1408.5302] [INSPIRE].

[41] A. Eichhorn, H. Gies, J. Jaeckel, T. Plehn, M.M. Scherer and R. Sondenheimer, The Higgs mass and the scale of new physics, JHEP 04 (2015) 022 [arXiv:1501.02812] [INSPIRE].

[42] F. Loebbert and J. Plefka, Quantum gravitational contributions to the standard model effective potential and vacuum stability, arXiv:1502.03093 [INSPIRE].

[43] Z. Lalak, M. Lewicki and P. Olszewski, Higher-order scalar interactions and SM vacuum stability, JHEP 05 (2014) 119 [arXiv:1402.3826] [INSPIRE].

[44] W. Israel, Singular hypersurfaces and thin shells in general relativity, Nuovo Cim. B 44 (1966) 4349.

[45] P. Bowcock, C. Charmousis and R. Gregory, General brane cosmologies and their global space-time structure, Class. Quant. Grav. 17 (2000) 4745 [hep-th/0007177] [INSPIRE].

[46] R. Gregory and A. Padilla, Nested brane worlds and strong brane gravity, Phys. Rev. D 65 (2002) 084013 [hep-th/0104262] [INSPIRE].

[47] A. Aguirre and M.C. Johnson, Dynamics and instability of false vacuum bubbles, Phys. Rev. D 72 (2005) 103525 [gr-qc/0508093] [INSPIRE].

[48] A. Aguirre and M.C. Johnson, Two tunnels to inflation, Phys. Rev. D 73 (2006) 123529 [gr-qc/0512034] [INSPIRE].

[49] G.W. Gibbons and S.W. Hawking, Action integrals and partition functions in quantum gravity, Phys. Rev. D 15 (1977) 2752 [InSPIRE].

[50] E. Witten, Anti-de Sitter space and holography, Adv. Theor. Math. Phys. 2 (1998) 253 [hep-th/9802150] [INSPIRE].

[51] F.A. Bais and R.J. Russell, Magnetic monopole solution of nonabelian gauge theory in curved space-time, Phys. Rev. D 11 (1975) 2692 [Erratum ibid. D 12 (1975) 3368] [InSPIRE].

[52] Y.M. Cho and P.G.O. Freund, Gravitating 't Hooft monopoles, Phys. Rev. D 12 (1975) 1588 [Erratum ibid. D 13 (1976) 531] [INSPIRE].

[53] G.W. Gibbons, Vacuum polarization and the spontaneous loss of charge by black holes, Commun. Math. Phys. 44 (1975) 245. 
[54] B.J. Carr and S.W. Hawking, Black holes in the early universe, Mon. Not. Roy. Astron. Soc. 168 (1974) 399 [INSPIRE].

[55] D.N. Page, Particle emission rates from a black hole: massless particles from an uncharged, nonrotating hole, Phys. Rev. D 13 (1976) 198 [InSPIRE].

[56] N. Arkani-Hamed, S. Dimopoulos and G.R. Dvali, The hierarchy problem and new dimensions at a millimeter, Phys. Lett. B 429 (1998) 263 [hep-ph/9803315] [INSPIRE].

[57] I. Antoniadis, N. Arkani-Hamed, S. Dimopoulos and G.R. Dvali, New dimensions at a millimeter to a Fermi and superstrings at a TeV, Phys. Lett. B 436 (1998) 257 [hep-ph/9804398] [INSPIRE].

[58] L. Randall and R. Sundrum, A large mass hierarchy from a small extra dimension, Phys. Rev. Lett. 83 (1999) 3370 [hep-ph/9905221] [INSPIRE].

[59] L. Randall and R. Sundrum, An alternative to compactification, Phys. Rev. Lett. 83 (1999) 4690 [hep-th/9906064] [INSPIRE].

[60] S. Dimopoulos and G.L. Landsberg, Black holes at the LHC, Phys. Rev. Lett. 87 (2001) 161602 [hep-ph/0106295] [INSPIRE].

[61] S.B. Giddings and S.D. Thomas, High-energy colliders as black hole factories: the end of short distance physics, Phys. Rev. D 65 (2002) 056010 [hep-ph/0106219] [INSPIRE].

[62] P. Kanti and E. Winstanley, Hawking radiation from higher-dimensional black holes, Fundam. Theor. Phys. 178 (2015) 229 [arXiv:1402.3952] [INSPIRE].

[63] R.C. Myers and M.J. Perry, Black holes in higher dimensional space-times, Annals Phys. 172 (1986) 304 [INSPIRE].

[64] P. Kanti, Black holes in theories with large extra dimensions: a review, Int. J. Mod. Phys. A 19 (2004) 4899 [hep-ph/0402168] [INSPIRE].

[65] R. Gregory, Braneworld black holes, Lect. Notes Phys. 769 (2009) 259 [arXiv:0804.2595] [INSPIRE].

[66] N. Dadhich, R. Maartens, P. Papadopoulos and V. Rezania, Black holes on the brane, Phys. Lett. B 487 (2000) 1 [hep-th/0003061] [INSPIRE].

[67] S.W. Hawking and G.T. Horowitz, The gravitational hamiltonian, action, entropy and surface terms, Class. Quant. Grav. 13 (1996) 1487 [gr-qc/9501014] [INSPIRE].

[68] R. Gregory and A. Padilla, Brane world instantons, Class. Quant. Grav. 19 (2002) 279 [hep-th/0107108] [INSPIRE].

[69] R. Emparan, G.T. Horowitz and R.C. Myers, Black holes radiate mainly on the brane, Phys. Rev. Lett. 85 (2000) 499 [hep-th/0003118] [INSPIRE].

[70] C.M. Harris and P. Kanti, Hawking radiation from a $(4+n)$-dimensional black hole: exact results for the Schwarzschild phase, JHEP 10 (2003) 014 [hep-ph/0309054] [INSPIRE]. 